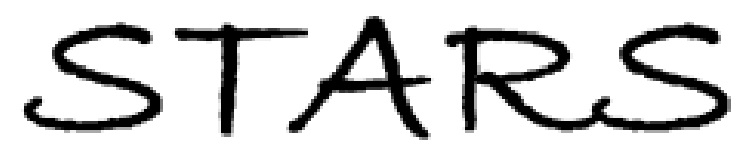

University of Central Florida

STARS

$1-1-2008$

\title{
Breaking of Symmetrical Periodic Solutions In A Singularly Perturbed Kdv Model
}

Alexander Tovbis

University of Central Florida

Find similar works at: https://stars.library.ucf.edu/facultybib2000 University of Central Florida Libraries http://library.ucf.edu

This Article is brought to you for free and open access by the Faculty Bibliography at STARS. It has been accepted for inclusion in Faculty Bibliography 2000 s by an authorized administrator of STARS. For more information, please contactSTARS@ucf.edu.

\section{Recommended Citation}

Tovbis, Alexander, "Breaking of Symmetrical Periodic Solutions In A Singularly Perturbed Kdv Model" (2008). Faculty Bibliography 2000s. 1066.

https://stars.library.ucf.edu/facultybib2000/1066

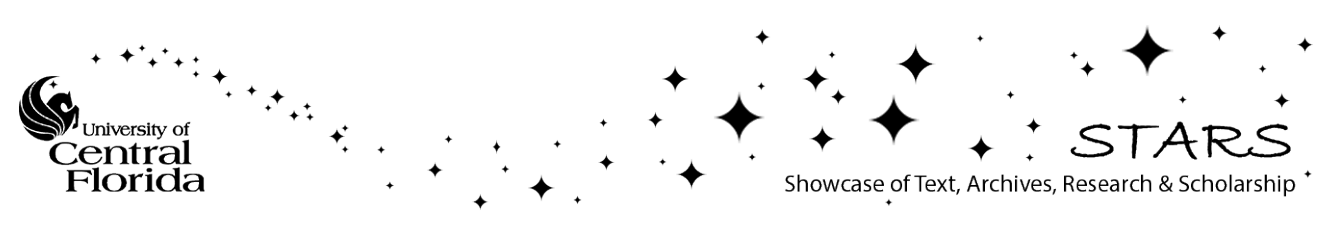




\title{
BREAKING OF SYMMETRICAL PERIODIC SOLUTIONS IN A SINGULARLY PERTURBED KDV MODEL*
}

\author{
ALEXANDER TOVBIS ${ }^{\dagger}$
}

\begin{abstract}
There are several recent developments in the well-known problem of breaking of homoclinic orbits (splitting of separatrices) of a system that undergoes a singular perturbation. First, survival of a homoclinic orbit is an exceptional situation that can be linked to triviality of the Stokes phenomenon of the underlying "truncated" equation. Second, homoclinic connections to exponentially small periodic orbits survive the perturbation in the generic case. In this paper we consider a different problem: we study deformations of "genuine" periodic orbits of the second order equation $y^{\prime \prime}=y+y^{2}$ that undergoes the singular perturbation $\varepsilon^{2} y^{\prime \prime \prime \prime}+\left(1-\varepsilon^{2}\right) y^{\prime \prime}=y+y^{2}$, where $\varepsilon>0$ is a small parameter. We prove that if the period and the constant of motion do not change too rapidly (in $\varepsilon$ ), a genuine (nontrivial) periodic solution does not survive the perturbation.
\end{abstract}

Key words. singular perturbations, periodic solutions, exponentially small phenomena

AMS subject classifications. 34E, 34M, 34D15, 37J

DOI. $10.1137 / 070694053$

\section{Introduction.}

1.1. Breaking of homoclinic connections. Let $y=0$ be a hyperbolic stationary point of an $n$-dimensional differential equation $y^{\prime}=f(y)$, and let $W_{s}, W_{u}$ be the stable and unstable manifolds at $y=0$. It is said that the stationary point $y=0$ has a homoclinic connection if there exists a phase trajectory, originating and ending at $y=0$, that lies on $W_{s} \cap W_{u}$. For example, the (bounded) separatrix solution

$$
y(x)=-\frac{3 / 2}{\cosh ^{2}(x / 2)}
$$

to

$$
y^{\prime \prime}(x)=y(x)+y^{2}(x)
$$

corresponds to the homoclinic connection of the stationary point $(0,0)$ in the phase plane of (2). There exists a general problem to describe how a singular perturbation of the original equation affects the homoclinic (or a heteroclinic) connection. A large number of particular singularly perturbed equations, originating from the corresponding physical or computational problems, have been discussed in the literature (see, for example, [STL] and the references therein). In particular, the singular perturbation

$$
\varepsilon^{2} y^{\prime \prime \prime \prime}(x)+\left(1-\varepsilon^{2}\right) y^{\prime \prime}(x)=y(x)+y^{2}(x)
$$

of (2) is related to the traveling wave reduction of a fifth order KdV equation that models gravity water waves. Existence or nonexistence of homoclinic connections of (3) has been studied in a number of papers; see, for example, [HM], [PRG], [GJ]. A rigorous proof of nonexistence of such connections was obtained in [AM] (see also

\footnotetext{
*Received by the editors June 8, 2007; accepted for publication (in revised form) June 4, 2008; published electronically November 19, 2008. This work was supported by NSF grant DMS 0508779. http://www.siam.org/journals/sima/40-4/69405.html

${ }^{\dagger}$ Department of Mathematics, University of Central Florida, Orlando, FL 32816 (atovbis@pegasus.cc.ucf.edu).
} 
[Eck]) and later on in [To4], where the "asymptotic beyond all orders" approach, first suggested in $[\mathrm{KS}]$ for a simple crystal growth model, was put on a rigorous basis. This approach is, in a sense, a natural way to study exponentially small phenomena, such as the mismatch between the stable and the unstable manifolds of (3). The goal of the present paper is to show that a similar argument can be used to prove nonexistence of symmetrical periodic solutions to (3) (see below), subject to some additional requirements.

The approach of $[\mathrm{KS}]$ is to rescale (3), which is called the outer equation, to the inner equation

$$
v^{\prime \prime \prime \prime}(z)+\left(1-\varepsilon^{2}\right) v^{\prime \prime}(z)=\varepsilon^{2} v(z)+v^{2}(z),
$$

where $x=\varepsilon z$ and $v=\varepsilon^{2} y$. The main advantage of (4) versus (3) is that the (exponentially small) difference between the stable and unstable solutions is detectable even in the leading order part

$$
v^{\prime \prime \prime \prime}(z)+v^{\prime \prime}(z)=v^{2}(z),
$$

of (4), which is called the truncated equation (4). This means that the difference between the stable and unstable solutions to (3) can be studied through the Stokes phenomenon of (5). In fact, in [TP] we considered the family of singular perturbations

$$
\varepsilon^{2} y^{(\mathrm{iv})}+\left(1-\varepsilon^{2}\right) y^{\prime \prime}-y=y^{2}+\varepsilon^{2} \gamma\left(2 y y^{\prime \prime}+\left(y^{\prime}\right)^{2}\right)
$$

of (2), parametrized by $\gamma \in \mathbb{R}$, and found exact conditions for persistence of the homoclinic connection (under the singular perturbation) in terms of the Stokes constant for the corresponding truncated inner equation. The technique developed in [To4] can be modified to consider singularly perturbed problems in other settings. For example, it was used in [To5] to show that the discretized equation (3) does not have a homoclinic connection.

Notice that persistence of the homoclinic connection under singular perturbations (3), (6) is equivalent to existence of a symmetrical (even) stable solution to the corresponding equation. Since the family of stable solutions to (3) (or to (6)) is one-dimensional (translation), we can arrange for $y^{\prime}(0)=0$; then the symmetry of the stable solution $y(x)$ is equivalent to $y^{\prime \prime \prime}(0)=0$.

1.2. Deformation of periodic solutions under singular perturbations. The problem of deformations of periodic solutions under singular perturbations has not yet received as much attention as the problem of homoclinic connections. However, it is known that there exist symmetrical periodic solutions to (3) that are exponentially small in $\varepsilon$; see [Lo] and later papers [IL1], [IL2]. We consider such periodic solutions as deformations of a constant solution $y \equiv 0$ of the unperturbed equation (2). Existence of even periodic solutions that are deformations of a trivial solution was also proved in [BPBA] for a certain class of reversible fourth order Hamiltonian systems with zero Hamiltonian. Discussion of even periodic solutions for some fourth order ODEs with nonzero Hamiltonian and cubic nonlinearity ((3) has a quadratic nonlinearity) can be found in $[\mathrm{PT}]$.

In the present paper we extended the technique of [To4] to study deformations of a genuine (nonconstant) periodic solution of (2). We limit our attention to periodic solutions inside the potential well of (2). Any such solution is given by the Weierstrass elliptic $\wp$-function with invariants $g_{2}, g_{3}$,

$$
y(x)=6 \wp_{g_{2}, g_{3}}\left(x-x_{0}\right)-\frac{1}{2},
$$


and has an integral of motion

$$
y^{\prime 2}=y^{2}+\frac{2}{3} y^{3}+C,
$$

where $x_{0} \in \mathbb{C}$ is a translation and $g_{2}=\frac{1}{12}, g_{3}=-\frac{1}{36}\left(C+\frac{1}{6}\right)$ (see section 1.3). The requirement $C \in\left(-\frac{1}{3}, 0\right)$ on the constant of motion (energy) $C$ guarantees that solution $y(x)$ is inside the potential well. (The value $C=0$ corresponds to the trivial solution and to the separatrix solution of (2), whereas $C=-\frac{1}{3}$ corresponds to the stationary solution $y(x) \equiv-1$.) Solution (7) is a periodic function with finite basic real and purely imaginary periods

$$
2 \omega_{1}=\int_{e_{1}}^{\infty} \frac{d u}{\sqrt{4 u^{3}-\frac{1}{12} u-g_{3}}}, \quad 2 \omega_{3}=i \int_{-\infty}^{e_{3}} \frac{d u}{\sqrt{4 u^{3}-\frac{1}{12} u-g_{3}}}
$$

where $e_{1}, e_{3}$ are the largest and the smallest real roots of $4 u^{3}-\frac{1}{12} u-g_{3}=0$ (see below), respectively. It is also an even function provided that $x_{0}$ is an integer linear combination of $\omega_{1}, \omega_{3}$. It will be convenient for us to choose

$$
x_{0}=\omega_{3},
$$

so that the unperturbed solution (7) is bounded, real-valued, symmetrical, and periodic with the period $2 \omega_{1}$ on $x \in \mathbb{R}$. Equivalently, we can consider solution (7) with $x_{0}=0$ along the horizontal line $\omega_{3}+\mathbb{R}$ in the complex $x$-plane.

The perturbed equation (3) also has an integral of motion

$$
\varepsilon^{2}\left(2 y^{\prime \prime \prime} y^{\prime}-y^{\prime \prime 2}\right)+\left(1-\varepsilon^{2}\right) y^{\prime 2}-y^{2}-\frac{2}{3} y^{3}=C(\varepsilon),
$$

where $C(\varepsilon)$ is the constant of motion (energy). Since we are interested in deformations of periodic solutions satisfying (8), we require $C(0)=C \in\left(-\frac{1}{3}, 0\right)$.

Let $\alpha \geq 1$. We say that a solution $y(x, \varepsilon)$ to the perturbed equation (3) is a $C^{\alpha}$-deformation of a periodic solution (7) (in the potential well) to the unperturbed equation (2) under the singular perturbation (3) on interval $S$ if

$$
Y(x, \varepsilon)=Y(x, 0)+\varepsilon^{\alpha} \tilde{Y}(x, \varepsilon),
$$

where vector $\tilde{Y}(x, \varepsilon)$ is continuous in $\varepsilon$, uniformly on interval $S$ of the $x$-axis. Here $Y(x, \varepsilon)=\operatorname{Col}\left(y(x, \varepsilon), y^{\prime}(x, \varepsilon), y^{\prime \prime}(x, \varepsilon), y^{\prime \prime \prime}(x, \varepsilon)\right)$. Note that a $C^{\alpha}$-deformation of a periodic solution (7) has constant of motion (11) satisfying

$$
C(\varepsilon)=C+\varepsilon^{\alpha} \tilde{C}(\varepsilon),
$$

where $\tilde{C}(\varepsilon)$ is a continuous function.

Our main result is the following theorem.

ThEOREM 1.1. Let $y(x, 0)=6 \wp_{\frac{1}{12}}, g_{3}\left(x-\omega_{3}\right)-\frac{1}{2}$, where $\left|g_{3}\right|<6^{-3}$, be a periodic solution (inside the potential well) of the nonperturbed equation (2). Let $n \in \mathbb{N}$ and $y(x, \varepsilon)$ be a $C^{\alpha}$-deformation, $\alpha \geq 1$, of $y(x, 0)$ under the perturbation (3) on some open interval $S$ that contains the segment $\left[\omega_{1}, n \omega_{1}\right]$. Then the deformation $y(x, \varepsilon)$ does not contain a sequence of symmetric periodic solutions $y\left(x, \varepsilon_{m}\right)$ of (3) with periods $2 k \omega_{1}\left(\varepsilon_{m}\right)$, where $k=1,2, \ldots, n, \lim _{m \rightarrow \infty} \varepsilon_{m}=0$, and $\omega_{1}(\varepsilon)$ is subject to

$$
\omega_{1}(\varepsilon)-\omega_{1}=\varepsilon^{\frac{\alpha}{2}} \tau(\varepsilon),
$$


where $\tau(\varepsilon)$ is a continuous function for small $\varepsilon \geq 0$.

Similarly to the homoclinic connection problem, the central idea of our proof here is uniform (in small $\varepsilon \geq 0$ ) control of an iterative solution of inner equation (4). In a certain sense, though, the homoclinic connection problem is simpler, because the manifolds of stable and unstable solutions to (3) are one-dimensional, whereas deformations of a periodic solution to (2) under the perturbation (3) form a manifold of the full dimension. We rescale the outer equation (3) to the inner equation (4) by $x=\varepsilon z, v=\varepsilon^{2} y$ and approach the problem through the following sequence of steps: (a) we construct by iterations a two-parameter family $\mathcal{F}_{\alpha}$ of deformations of the rescaled periodic solution (7), (10), satisfying (4); (b) we show that solutions $v(z, \varepsilon)$ to the inner equation (4) that corresponds to a $C^{\alpha}$-deformation $y(x, \varepsilon)$ of a periodic solution (7) with the period $2 \omega_{1}$ belong to the family $\mathcal{F}_{\alpha}$; and (c) for any $k \in \mathbb{N}$ we prove that the family $\mathcal{F}_{\alpha}$ does not contain a sequence $\left\{v\left(z, \varepsilon_{m}\right)\right\}_{1}^{\infty}$ of symmetrical and periodic (with the period $\left.2 k \frac{\omega_{1}\left(\varepsilon_{m}\right)}{\varepsilon_{m}}\right)$ solutions if $\omega_{1}(\varepsilon)$ is subject to (14).

The paper is organized in the following way. In the remaining part of this section we describe the Stokes phenomenon for the truncated inner equation (5) and some connections between deformations of homoclinic and periodic orbits of (3). Solution of (3) by iterations, which yields the two-parameter family of solutions $\mathcal{F}_{\alpha}$, is obtained in sections 2-3. Finally, the proof of Theorem 1.1 is completed in section 4 .

1.3. Periodic solutions of the unperturbed equation. It is easy to check that the values $C$ from the interval $\left(-\frac{1}{3}, 0\right)$ define periodic solutions of the unperturbed equation (2) within the well of the potential $-y^{2}-\frac{2}{3} y^{3}$. After the change of variables $y=6 X-\frac{1}{2},(8)$ is reduced to

$$
X^{\prime 2}=4 X^{3}-\frac{1}{12} X-g_{3}
$$

where $g_{3}=-\frac{1}{36}\left(C+\frac{1}{6}\right)$. The solution to this equation is given by the Weierstrass elliptic function $\wp(x)=\wp_{g_{2}, g_{3}}(x)$, where the invariant $g_{2}=\frac{1}{12}$ and $g_{3}$ is defined above. Thus, periodic solutions to the unperturbed equation (2) are given by

$$
y(x)=6 \wp_{g_{2}, g_{3}}(x)-\frac{1}{2} .
$$

The number $\Delta=g_{2}^{3}-27 g_{3}^{2}$ is called the discriminant of $\wp_{g_{2}, g_{3}}(x)$. The condition $\Delta>0$ is equivalent to $\left|g_{3}\right|<6^{-3}$, i.e., to $C \in\left(-\frac{1}{3}, 0\right)$. Under this condition the Weierstrass function $\wp$ has real period $2 \omega_{1}$ defined by (9) and purely imaginary period $2 \omega_{3}$, where $\omega_{1},-i \omega_{3}$ are positive numbers (the latter requires correct choice of the branch of the square root in (9)). Using the standard notation, we denote $\omega_{2}=\omega_{1}+\omega_{3}$ and $e_{j}=\wp\left(\omega_{j}\right)$, where $j=1,2,3$. It is well known that (see, for example, [GR]) $e_{j}$ are the roots of the cubic polynomial in (15),

$$
\wp^{\prime}\left(\omega_{j}\right)=0, j=1,2,3, \quad e_{1}+e_{2}+e_{3}=0, \quad \text { and } \quad e_{3} \leq e_{2} \leq e_{1} .
$$

When $x$ varies along the real axis, the value of $\wp$ varies between $e_{1}$ and $+\infty$ (as $\wp(x)$ is an even function that has a second order pole at the origin with the principle part $\left.\frac{1}{x^{2}}\right)$; see Figure 1 .

This corresponds to the periodic solution of (2), defined by (16), with the range from $\tilde{e}_{1}$ to infinity outside the potential well, i.e., to the unbounded branch of the periodic orbit (the energy of the motion is fixed). Here $\tilde{e}_{j}=6 e_{j}-\frac{1}{2}, j=1,2,3$; see Figure 2 . 


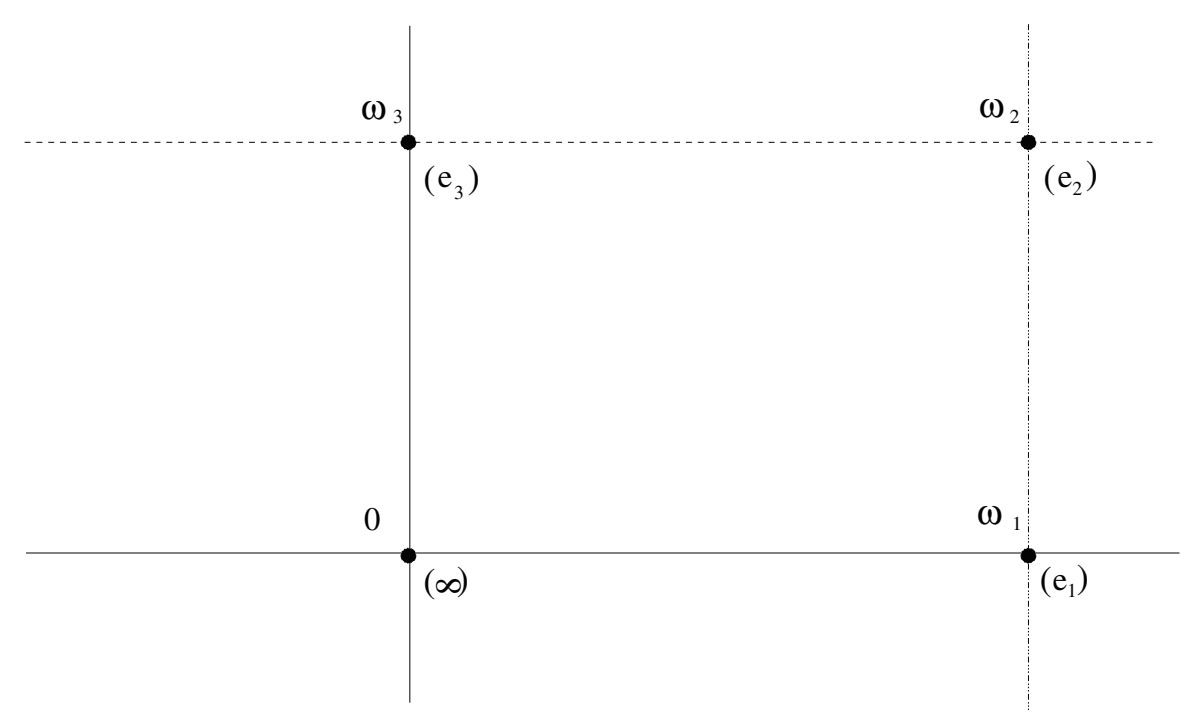

FIG. 1. Part of the parallelogram of periods $0 \omega_{1} \omega_{2} \omega_{3}$ for the Weierstrass functions $\wp(x)$ in the complex $x$-plane together with the corresponding values of $\wp$ shown in the brackets.

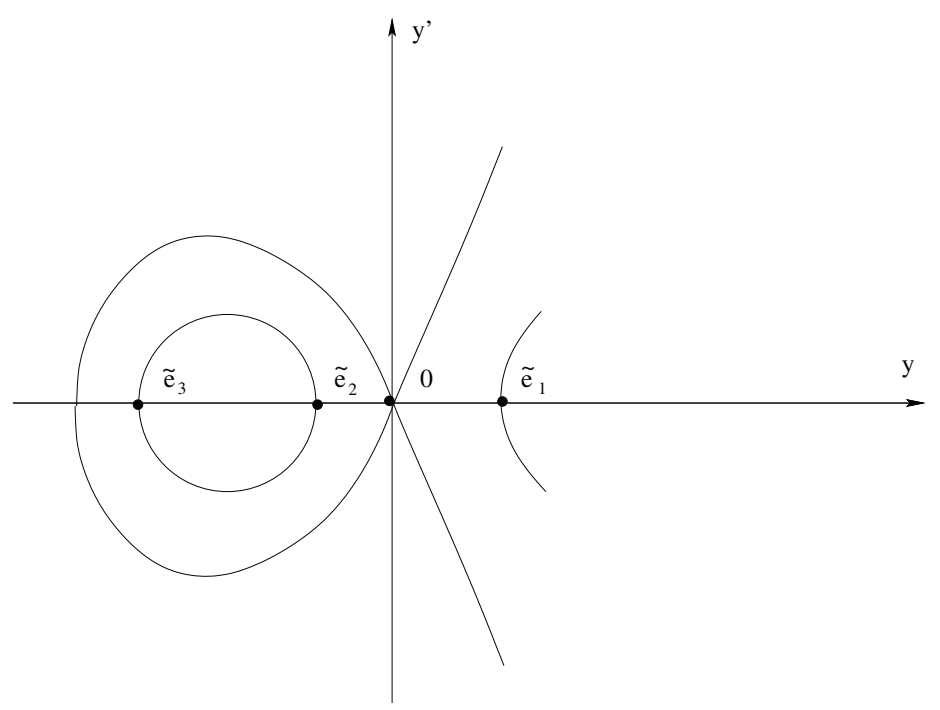

FIG. 2. Phase portrait of (2) showing periodic solutions $(\Delta>0)$ of the same period inside (bounded) and outside (unbounded) the potential well. Here $\tilde{e}_{j}=6 e_{j}-\frac{1}{2}, j=1,2,3$.

When $x$ varies along the horizontal line $\Im x=\omega_{3}$, the value of $\wp$ varies between $e_{3}$ and $e_{2}$. That corresponds to the periodic solution of (2), defined by (16), with the range from $\tilde{e}_{3}$ to $\tilde{e}_{2}$ inside the potential well, i.e., to the bounded branch of the periodic orbit. When $x$ varies along the vertical line $\Re x=\omega_{1}$, the value of $\wp$ varies between $e_{1}$ and $e_{2}$. That corresponds to the tunneling between the unbounded and bounded branches of the periodic orbit defined by the solution (16); see Figures 1 and 2. For $\Delta<0$ the periods $\omega_{1}$ and $\omega_{3}$ are complex conjugated so that $\omega_{2}$ is real. In this case the value of $\wp$ varies between $e_{2}$ and $+\infty$, which corresponds to infinite periodic motion outside the potential well; see Figure 3. 


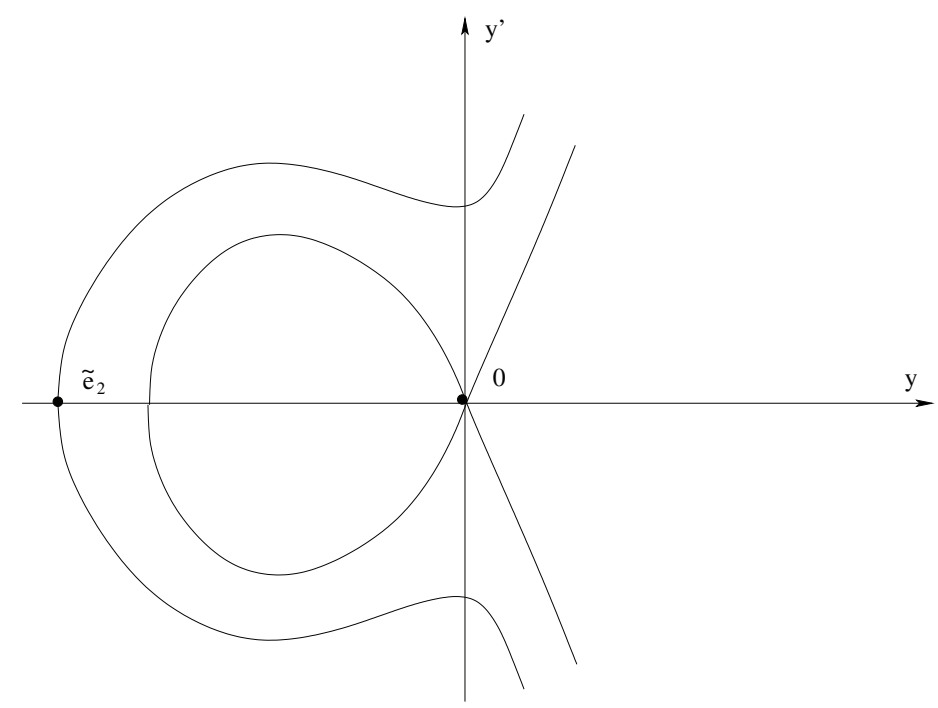

FIG. 3. Phase portrait of (2) showing an unbounded periodic solution $(\Delta<0)$ outside the potential well. Here $\tilde{e}_{2}=6 e_{2}-\frac{1}{2}$.

Here and henceforth it will be convenient for us to consider $x_{0}$ from (7) to be zero. Then we are interested in the behavior of $y(x)$ along the horizontal line $\omega_{3}(\varepsilon)+\mathbb{R}$.

1.4. Solutions to the perturbed equation. Considering perturbations of the separatrix solution (1) of the original equation, we focus our attention on the stable and unstable solutions of the perturbed equation (3) (those are solutions corresponding to the one-dimensional manifolds $W_{s}$ and $W_{u}$ in the phase space of (3)). We define the unstable and stable solutions to (3) as

$$
y_{u, s}(x, \varepsilon)=\sum_{k=1}^{\infty} y_{k}(\varepsilon) e^{ \pm k x}
$$

respectively, where $y_{1}(\varepsilon)$ as an arbitrary continuous positive function and all $y_{k}, k>1$, are uniquely defined through $y_{1}$ [To4]. The series (18) is convergent in properly chosen left- and right-half planes of the complex $x$-plane, respectively. It is easy to check that for $\varepsilon=0$ the choice of $y_{1}(0)=6$ yields the unbounded separatrix solution

$$
y(x)=\frac{3 / 2}{\sinh ^{2}(x / 2)},
$$

which is related to the bounded separatrix solution (1) through the shift $x \mapsto x+i \pi$. Considering $x$ as a complex variable, now and henceforth we refer to (19) as the separatrix solution of (3).

Perturbations of periodic solutions to the original equation (2) that we are interested in are not as clearly identifiable as solutions (18) in the separatrix case. We start with considering the separatrix solution of (3) as the limit of periodic solutions (16) as $C \rightarrow 0^{-}$. In this limit the real half-period $\omega_{1} \rightarrow \infty$, and the imaginary half-period $\omega_{3} \rightarrow i \pi$. Thus, the point $\omega_{2} \rightarrow i \pi+\infty$ as $C \rightarrow 0^{-}$.

Note that the stable and unstable solutions (18) are symmetrical with respect to $x=0$ since $y_{s}(x, \varepsilon)=y_{u}(-x, \varepsilon)$. Alternatively, we can consider $y_{u}(x)$ as the 
symmetrical continuation of $y_{s}(x)$ through $x=\infty$ on the compactification of $\mathbb{R}$. The same observation is correct for the horizontal line $\Im x=\pi$ and $x=i \pi+\infty$. The analogue of the point $x=i \pi+\infty$ in the periodic case is $x=\omega_{2}$. Therefore, by analogy with the separatrix case, we will consider perturbations of the periodic solution (16) that are symmetrical with respect to the point $\omega_{2}(\varepsilon)$. Equivalently, that means that the first and third derivatives of $y(x)$ turn zero at $x_{0}=\omega_{2}$. The remaining two of the total of four conditions to define $y(x, \varepsilon)$ are $y^{\prime}\left(\omega_{3}(\varepsilon), \varepsilon\right)=0$ and the integral of motion (11). Thus, $y(x, \varepsilon)$ satisfies third order differential equation (11) with the prescribed energy $C(\varepsilon)$ together with three boundary conditions

$$
y^{\prime}\left(\omega_{3}(\varepsilon), \varepsilon\right)=0, \quad y^{\prime}\left(\omega_{2}(\varepsilon), \varepsilon\right)=y^{\prime \prime \prime}\left(\omega_{2}(\varepsilon), \varepsilon\right)=0,
$$

where $C(0)=C$ and $2 \omega_{1}(\varepsilon), 2 \omega_{3}(\varepsilon)$ are the fundamental periods of the Weierstrass function $\wp$ in the solution (16) of (8).

It is easy to see that if $f(x)$ is an even (symmetrical at $x=0$ ) periodic function with a period $2 \omega_{1}$, then $f(x)$ is symmetrical at any point $x=k \omega_{1}, k \in \mathbb{N}$. Since a solution $y(x, \varepsilon)$ to the BVP $(11),(20)$ is symmetrical at $x=\omega_{2}(\varepsilon)$ on the horizontal line $\Im x=\Im \omega_{3}(\varepsilon), y(x, \varepsilon)$ is an even periodic solution if and only if

$$
y^{\prime \prime \prime}\left(\omega_{3}(\varepsilon), \varepsilon\right)=0 .
$$

1.5. The Stokes phenomenon for the inner equation. Periodic solutions (16) to (2) have second order poles at $x=0$. The asymptotic beyond all orders approach of $[\mathrm{KS}]$ suggests blowing up this singularity by rescaling

$$
x=\varepsilon z, \quad y(x, \varepsilon)=\varepsilon^{-2} v(z, \varepsilon),
$$

where $z$ is a new independent complex variable. The rescaled equation (inner equation) is given by (4), which we can rewrite as

$$
\left(D_{z}^{2}+1\right)\left(D_{z}^{2}-\varepsilon^{2}\right) v=v^{2} .
$$

Let $v_{+}(z, \varepsilon)$ and $v_{-}(z, \varepsilon)$ denote, respectively, the rescaled solutions to the BVP $(11),(20)$ and to its "symmetrical" problem, where conditions at $\omega_{2}(\varepsilon)$ are replaced by the same conditions at $-\bar{\omega}_{2}(\varepsilon)=-\omega_{1}(\varepsilon)+\omega_{3}(\varepsilon)$, i.e., $y^{\prime}\left(-\bar{\omega}_{2}(\varepsilon), \varepsilon\right)=y^{\prime \prime \prime}\left(-\bar{\omega}_{2}(\varepsilon), \varepsilon\right)=$ 0 . Our observation (proved below) is that, similarly to the case of the separatrix solution, the leading order (in $\varepsilon$ ) of the difference between $v_{+}(z, \varepsilon)$ and $v_{-}(z, \varepsilon)$ can be derived from the truncated equation

$$
\left(D_{z}^{2}+1\right) D_{z}^{2} v=v^{2} .
$$

Note that $z=\infty$ is the irregular singular point of (24) and that this equation has a unique formal power series solution

$$
\hat{v}(z)=\sum_{k=1}^{\infty} \frac{v_{k}}{z^{2 k}}
$$

where $v_{1}=6$.

The inverse Laplace transform $\mathcal{L}^{-1}$ converts the truncated inner equation (24) into the convolution equation

$$
\left(p^{4}+p^{2}\right) V(p)=V(p) * V(p),
$$


where $p$ is a dual variable (called Borel variable), $V(p)=\left[\mathcal{L}^{-1} v\right](p)$, and $F(p) * G(p)=$ $\int_{0}^{p} F(p-\tau) G(\tau) d \tau$. It is well known that the asymptotic expansion (25) of $v(z)$ yields the corresponding asymptotic expansion in powers of $p$ of $V(p)$ at $p=0$.

THEOREM 1.2. Equation (26) admits a unique nontrivial power series solution in odd powers of $p$. This solution defines a function $V(p)$ that is analytic at the whole $p$-plane except for two vertical rays: from $p=i$ upward and from $p=-i$ downward. The function $V(p)$ is of exponential order 1 along any nonvertical ray in this cut p-plane.

This theorem follows from a more general statement (Main Theorem) of [To2]. Corollary 1.3. Let $v_{ \pm}(z)$ be defined by

$$
v_{ \pm}(z)=\int_{0}^{ \pm \infty} e^{-z p} V(p) d p
$$

These functions are the only analytic solutions of the truncated inner equation (24) that satisfy

$$
v_{ \pm}(z) \sim \hat{v}(z) \quad \text { as } z \rightarrow \infty, \quad z \in S_{ \pm}
$$

where $S_{ \pm}$are sectors

$$
S_{+}=\{z:|\arg z|<\pi\}, \quad S_{-}=\{z:|\arg z-\pi|<\pi\} .
$$

Moreover,

$$
v_{+}(z)-v_{-}(z)=-2 \pi i s e^{i z}(1+o(1)) \quad \text { as } \quad z \rightarrow \infty, \quad 0<\arg z<\pi,
$$

where the constant $s$ is determined through

$$
s=\lim _{p \rightarrow-i}(p+i) V(p) .
$$

Proof. The Taylor expansion of $V(p)$ at $p=0$ can be obtained by applying the inverse Laplace transform (Borel transform) to the formal series $\hat{v}(z)$. The function $V(p)$ is analytic on a Riemann surface that has possible branch points of the logarithmic type at $p=i k$, where $k \in \mathbb{Z} \backslash\{0\}$. The statement on the behavior of $V(p)$ at singularities $p= \pm i$ follows from Theorem 2.2 in [To2]. The uniqueness of solutions $v_{ \pm}(z)$ satisfying (28) follows from Theorem 1.2 and properties of the Laplace transform.

Definition 1.4. The constant $s$ in (31) is called the Stokes constant for the truncated inner equation (24).

PROPOSITION 1.5. The following three conditions are equivalent: (i) the formal power series solution (25) has a positive radius of convergence; (ii) $s=0$; and (iii) $v_{+}(z) \equiv v_{-}(z)$.

Proof. The fact that (i) implies (iii) is obvious. The inverse statement follows from the fact that $v(z)=v_{+}(z) \equiv v_{-}(z)$ implies that the function $v(z)$ is singlevalued near infinity and has asymptotic expansion $\hat{v}(z)$ in the full neighborhood of infinity (see [Wa]). It is also clear that (i) implies (ii), since in this case $\mathcal{L}^{-1} \hat{v}(z)$ is an entire function. Suppose now that $s=0$. Since $V(p)$ is real-analytic on $\mathbb{R}$, we obtain that $V(p)=o\left(\frac{1}{p-( \pm i)}\right)$ as $p \rightarrow \pm i$. That means, according to Corollary 2.2 in [To2], that $v_{+}(z)$ coincides with $v_{-}(z)$ in both the lower and upper $z$ half-planes. Thus (ii) implies (iii). 
Divergence (zero radius of convergence) of the formal power series (25) was proved in [To2, p. 247]. According to Proposition 1.5, this implies that $v_{+}(z) \not \equiv v_{-}(z)$. (In fact, nonzero numerical values of $s$ for equations, equivalent to (24), were computed in [PRG], [GJ] by means of formal Borel summation.) This implies, at least at the formal level, that solution $v_{+}(z, \varepsilon)$ to $(23)$ does not coincide with solution $v_{-}(z, \varepsilon)$ to (23). To make this statement rigorous, it is sufficient to show that (a)

$$
v_{ \pm}(z, \varepsilon) \rightarrow v_{ \pm}(z)
$$

as $\varepsilon \rightarrow 0$ in some regions $R_{ \pm}$uniformly in $z \in R_{ \pm}$, and (b) intersection $R_{+} \cap R_{-}$ contains some segment of a positive length.

2. Inner equation. In the following theorem (Theorem 2.1) we construct a two-parameter family of solutions to $(23)$ that are symmetrical at $z=\tilde{\omega}_{2}(\varepsilon)$ and that converge to $v_{+}(z)$, or to its translation $v_{+}(z-h), h \in \mathbb{R}$, as $\varepsilon \rightarrow 0$ uniformly in the corresponding region (see below) of the complex $z$-plane. Here $\tilde{\omega}_{j}(\varepsilon)=\frac{\omega_{j}(\varepsilon)}{\varepsilon}$, $j=1,2,3$.

This result constitutes an essential part of the method of $[\mathrm{KS}]$; convergence of $v_{+}(z, \varepsilon)$ to $v_{+}(z)$ was mentioned only briefly in the original paper [KS] (with regard to a third order equation considered there), but explicit formulations and proofs were omitted. In other papers, connection between solutions of (23) and (24) was considered only on the formal level. The proof of convergence of $v_{+}(z, \varepsilon)$ to $v_{+}(z)$ in the separatrix case was given in [To4] (see also [To5] for the discretized equation (2), as well as [TP] for a more general family of singular perturbations of (2)). The major difficulty there was based on the fact that solutions of the full and of the truncated inner equations have different rates of convergence to 0 as $z \rightarrow \infty$ (in proper directions): $v_{+}(z, \varepsilon), \varepsilon>0$, approaches 0 exponentially fast (in $z$ ), while $v_{+}(z)$ has only power order convergence. One needs to find, however, some uniform majorization that will cover both cases. In order to find such a majorization, we suggested [To4], [To5] linearizing (23) around the unperturbed solution $6 q^{2}$, where $q(z, \varepsilon)=\frac{\varepsilon}{\sinh \frac{\varepsilon z}{2}}$, and applying the contraction mapping principle to the obtained equation. A similar approach will be developed below with the natural choice

$$
q(z, \varepsilon)=\varepsilon \sqrt{\wp_{\frac{1}{12}, g_{3}(\varepsilon)}(\varepsilon z)-\frac{1}{12}}
$$

where $g_{3}=g_{3}(\varepsilon)$ is determined through $\omega_{1}=\omega_{1}(\varepsilon)$ by $(9)$.

2.1. Linearized equation. The substitution

$$
v(z, \varepsilon)=u(z, \varepsilon)+6 c(\varepsilon) q^{2}(z, \varepsilon),
$$

where $q(z, \varepsilon)$ is given by (33), reduces (23) to

$$
\left(D_{z}^{2}+1\right)\left(D_{z}^{2}-\varepsilon^{2}\right) u=u^{2}+12 c(\varepsilon) q^{2} u+f(q) .
$$

Here $c=c(\varepsilon)$ is a continuous function satisfying $c(0)=1$. Calculations of $f$ after some algebra yield

$$
f(q)=c\left[36\left(c-1-4 \varepsilon^{2}\right) q^{4}-6 ! q^{6}-2 \varepsilon^{6} J(\varepsilon)\right],
$$

where

$$
J(\varepsilon)=-36 g_{3}(\varepsilon)-\frac{1}{6}
$$


is determined through $\omega_{1}=\omega_{1}(\varepsilon)$. (To simplify notation, we omit the $\varepsilon$ dependence in $\omega_{j}$ or $\tilde{\omega}_{j}, j=1,2,3$, provided that such omission cannot cause any misunderstanding.) Note that $\lim _{\varepsilon \rightarrow 0} 6 c(\varepsilon) q^{2}(z, \varepsilon)=\frac{6}{z^{2}}$. This is the leading term of the asymptotic expansion (25) of $v_{+}(z)$. So, substitution (34) linearizes (23) with respect to the leading term for both positive and zero values of $\varepsilon$.

Let

$$
w=\left(D^{2}+1\right) u
$$

Then (35) becomes

$$
\left(D^{2}-\varepsilon^{2}\right) w=\left[\left(D^{2}+1\right)^{-1} w\right]^{2}+12 c q^{2}\left(D^{2}+1\right)^{-1} w+f(q)
$$

or

$$
\begin{aligned}
{\left[D^{2}-\left(\varepsilon^{2}+12 q^{2}\right)\right] w=} & {\left[\left(D^{2}+1\right)^{-1} w\right]^{2} } \\
& +12 c q^{2}\left[\left(D^{2}+1\right)^{-1}-1\right] w-12 q^{2}(1-c) w+f(q) .
\end{aligned}
$$

Using the identity

$$
\left(D^{2}+1\right)^{-1}-1=-\left(D^{2}+1\right)^{-1} D^{2}
$$

and solving (39) for $D^{2} w$, we finally get

$$
\begin{aligned}
& {\left[D^{2}-\left(\varepsilon^{2}+12 q^{2}\right)\right] w=\left[1-12 c q^{2}\left(D^{2}+1\right)^{-1}\right]\left(f(q)+\left[\left(D^{2}+1\right)^{-1} w\right]^{2}\right)} \\
& -12 q^{2}(1-c) w-12 c q^{2}\left(D^{2}+1\right)^{-1}\left[\varepsilon^{2}+12 c q^{2}\left(D^{2}+1\right)^{-1}\right] w .
\end{aligned}
$$

This integrodifferential equation is equivalent to (35), (38). We solve (42) by the contraction mapping principle, considering it as a "perturbation" of the nonhomogeneous linear ODE

$$
\left[D^{2}-\left(\varepsilon^{2}+12 q^{2}\right)\right] w=\left[1-12 c q^{2}\left(D^{2}+1\right)^{-1}\right] f(q) .
$$

The corresponding homogeneous equation

$$
\left[D^{2}-\left(\varepsilon^{2}+12 q^{2}\right)\right] w=0
$$

has two independent solutions $v_{1}(z)$ and $v_{2}(z)$, where $v_{2}(z)$ is symmetric (even) and $v_{1}(z)$ is antisymmetric (odd) at the origin. Indeed, $v=6 q^{2}$ is a solution to $\left(D^{2}-\varepsilon^{2}\right) v=$ $v^{2}$, which is the rescaled equation (2). Differentiating both sides of the latter equation, we get $\left(D^{2}-\varepsilon^{2}\right) v^{\prime}=2 v v^{\prime}$. This equation coincides with (44), where $w=v^{\prime}$. Thus, we obtain

$$
v_{1}(z)=6 \frac{d}{d z} q^{2}=6 \varepsilon^{3} \wp^{\prime}(\varepsilon z) .
$$

The second linearly independent solution can be taken as

$$
v_{2}(z)=v_{1}(z) \int_{0}^{z} \frac{d \xi}{v_{1}^{2}(\xi)} .
$$

Copyright (C) by SIAM. Unauthorized reproduction of this article is prohibited. 
Note that $\wp(\varepsilon z)$ is symmetrical and, thus, $v_{1}$ is an antisymmetrical (odd) function with respect to any integer combination of half periods $\tilde{\omega}_{1}$ and $\tilde{\omega}_{3}$. It will be shown below (section 2.3) that solutions

$$
v_{2 j}(z)=v_{2}(z)-\frac{1}{6 \varepsilon^{7} \Delta}\left[\frac{3 g_{3}}{2} \omega_{j}-g_{2} \zeta\left(\omega_{j}\right)\right] v_{1}(z), \quad j=1,2,3,
$$

to (44), where

$$
\zeta(x)=\frac{1}{x}-\int_{0}^{x}\left(\wp(y)-\frac{1}{y^{2}}\right) d y
$$

is a "negative antiderivative" of $\wp$, are symmetrical at the points $z=\tilde{\omega}_{j}$, respectively.

2.2. Formulation of the theorem. Let $g(z)$ be a function, symmetrical (even) with respect to the point $\tilde{\omega}_{2}(\varepsilon)$, and let $\mathcal{I}_{2}=\left(D^{2}+1\right)^{-1}$ denote the operator, inverse to $D^{2}+1$ and such that $\mathcal{I}_{2} g(z)$ is symmetrical with respect to $\tilde{\omega}_{2}(\varepsilon)$. The construction of $\mathcal{I}_{2}$ will be given in section 3.1 .

Equation (42) can be written in the operator form as $w=\mathcal{N} w$, where

$$
\mathcal{N} w=\mathcal{I}_{1} \circ\left[\left(1-12 c q^{2} \mathcal{I}_{2}\right)\left[f+\left(\mathcal{I}_{2} w\right)^{2}\right]-12 q^{2}(1-c) w+12 c q^{2} \mathcal{I}_{2}\left(\varepsilon^{2}+12 c q^{2} \mathcal{I}_{2}\right) w\right]
$$

and

$$
\mathcal{I}_{1} g(z)=-v_{1}(z) \int_{\tilde{\omega}_{2}}^{z} v_{2}(\xi) g(\xi) d \xi+v_{2}(z) \int_{\tilde{\omega}_{2}}^{z} v_{1}(\xi) g(\xi) d \xi .
$$

We define iterations

$$
w_{0}=0, \quad w_{1}=\mathcal{N} w_{0}+\varepsilon^{6} b(\varepsilon) v_{22}(z), \quad w_{n}=\mathcal{N} w_{n-1}, n=2,3, \ldots,
$$

and $\Delta w_{n}=w_{n}-w_{n-1}, n=1,2, \ldots$, where $b(\varepsilon)$ is a continuous function in a vicinity of $\varepsilon=0$. The following theorem proves uniform convergence of the series $\sum_{n=1}^{\infty} \Delta w_{n}$ in a region $R_{z_{0}} \subset \mathbb{C}$, which is defined below. Thus, we obtain a family of solutions to (42), parametrized by $c(\varepsilon)$ and $b(\varepsilon)$, which are symmetrical at $z=\tilde{\omega}_{2}$.

Consider the parallelogram of periods of the elliptic function $q(z, \varepsilon)$ (the periods are $2 \tilde{\omega}_{1}$ and $\left.2 \tilde{\omega}_{3}\right)$ centered at the origin with the square cut that has vertices $\pm z_{0}(1+i)$ and $\pm z_{0}(1-i)$, where $z_{0}>0$ is a positive constant. We require $\varepsilon \in\left[0, \varepsilon_{0}\right]$, where

$$
\varepsilon_{0} z_{0}<\min \left\{\omega_{1},\left|\omega_{3}\right|\right\}
$$

By $R_{z_{0}}$ we denote a quarter of this figure, i.e., of the parallelogram of periods with the square cut, that lies in the first quadrant; see Figure 4.

TheOREM 2.1. Let $E$ be a closed subinterval of $\left(-\frac{1}{3}, 0\right)$, and let $J(\varepsilon)$ from $(37)$ be a function, defined on a neighborhood of $\varepsilon=0$ with the range in $E$. Let continuous functions $b(\varepsilon), c(\varepsilon)$ satisfy

$$
|b(\varepsilon)| \leq l \varepsilon^{\alpha} \quad \text { and } \quad|c(\varepsilon)-1| \leq l \varepsilon^{\alpha}
$$

for some $l>0, \alpha \geq 1$, and for all nonnegative $\varepsilon$ from a vicinity of $\varepsilon=0$. Then there exist $\varepsilon_{0}>0, z_{0}>0$ satisfying (52), which depend only on $E$ and $l$, and such that the series

$$
w(z, \varepsilon)=\sum_{n=1}^{\infty} \Delta w_{n}(z, \varepsilon)
$$

Copyright $@$ by SIAM. Unauthorized reproduction of this article is prohibited. 


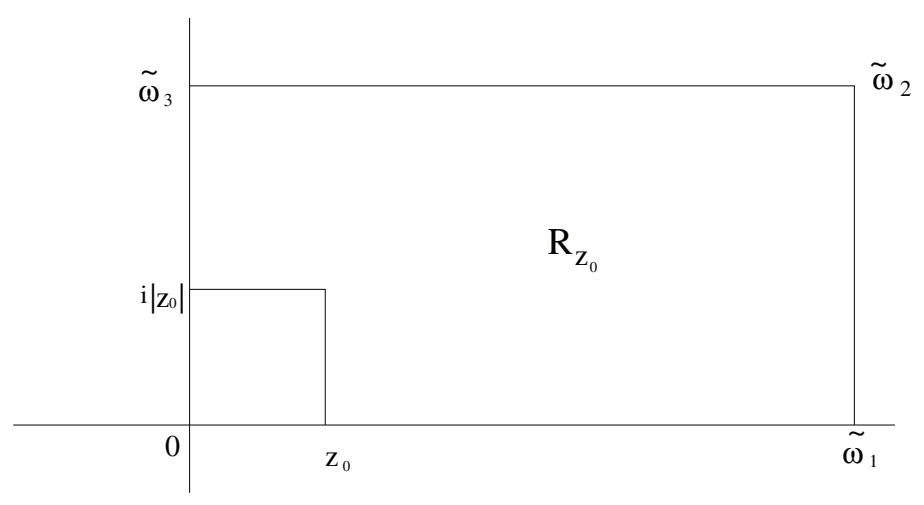

FIG. 4. Region $R_{z_{0}}$.

converges uniformly in $R_{z_{0}} \times\left[0, \varepsilon_{0}\right]$.

Remark 2.2. The rectangular "cut" in $R_{z_{0}}$ can be replaced by a cut of any other shape so that the distance between $R_{z_{0}}$ and the origin is $O\left(z_{0}\right)$. In fact, it will be replaced by a more convenient triangular "cut" in the course of the proof.

The proof of the theorem is given in section 3.4.

2.3. The second fundamental solution to the linearized equation. Note that, similarly to the separatrix case, the solution $v_{1}$ to (44) is an odd function, while the solution $v_{2}$ is an even function; see (45)-(46). Moreover, $\wp(\varepsilon z)$ is symmetrical, and, thus, $v_{1}$ is an antisymmetrical (odd) function with respect to any integer combination of half-periods $\tilde{\omega}_{1}$ and $\tilde{\omega}_{3}$.

Our computation of $v_{2}$ is based on the well-known fact (see, for example, [WW]) that the difference between two elliptic functions with the same periods and same principal parts at each singular point is a constant. The only zeros of the function $\wp^{\prime}(\varepsilon z)$ within the parallelogram of periods are simple zeros at the points $\tilde{\omega}_{j}, j=1,2,3$. Therefore

$$
\frac{1}{\wp^{\prime 2}(\varepsilon z)}=A_{0}+\sum_{j=1}^{3} A_{j} \wp\left(\varepsilon z-\omega_{j}\right)
$$

for all $z$ and

$$
\int_{0}^{z} \frac{d \xi}{{\wp^{\prime}}^{2}(\varepsilon \xi)}=A_{0} z-\frac{1}{\varepsilon} \sum_{j=1}^{3} A_{j}\left[\zeta\left(\varepsilon z-\omega_{j}\right)+\zeta\left(\omega_{j}\right)\right],
$$

where the constants $A_{k}, k=0,1,2,3$, will be discussed below and $\zeta(x)$, defined by (48), is an odd function. The following arguments use some standard facts about the Weierstrass $\wp$-function that can be found, for example, in [WW], [Ha].

Using the "addition theorem" for $\zeta$-function

$$
\zeta\left(\varepsilon z-\omega_{j}\right)-\zeta\left(-\omega_{j}\right)=\zeta(\varepsilon z)+\frac{1}{2} \frac{\wp^{\prime}(\varepsilon z)}{\wp(\varepsilon z)-e_{j}}, \quad j=1,2,3,
$$

we get

$$
\int_{0}^{z} \frac{d \xi}{\wp^{\prime 2}(\varepsilon \xi)}=\frac{1}{\varepsilon}\left[A_{0} \varepsilon z-\zeta(\varepsilon z) \sum_{j=1}^{3} A_{j}-\frac{1}{2} \wp^{\prime}(\varepsilon z) \sum_{j=1}^{3} \frac{A_{j}}{\wp(\varepsilon z)-e_{j}}\right] .
$$


Constants $A_{k}, k=0,1,2,3$, are calculated in Lemma 2.3 below. Differential equations

$$
\wp^{\prime 2}=4 \prod_{j=1}^{3}\left(\wp-e_{j}\right) \quad \text { and } \quad \wp^{\prime \prime}=6 \wp^{2}-\frac{g_{2}}{2}
$$

for the Weierstrass $\wp$-function and identities

$$
e_{1} e_{2}+e_{1} e_{3}+e_{2} e_{3}=-\frac{g_{2}}{4} \quad \text { and } \quad e_{1} e_{2} e_{3}=\frac{g_{3}}{4}
$$

are also utilized in this lemma.

LEMMA 2.3.

$$
A_{0}=\frac{9 g_{3}}{\Delta}, \quad A_{j}=\left[\frac{1}{2\left(e_{j}-e_{i}\right)\left(e_{j}-e_{k}\right)}\right]^{2}, \quad j=1,2,3, \quad \text { and } \quad A_{+}=\sum_{j=1}^{3} A_{j}=\frac{6 g_{2}}{\Delta},
$$

where the indices $i, j, k$ are a permutation of $1,2,3$.

Proof. The Taylor expansion of $\wp$ at $\omega_{j}, j=1,2,3$, yields

$$
\wp(x)=e_{j}+a_{j}\left(x-\omega_{j}\right)^{2}+O\left(x-\omega_{j}\right)^{4} .
$$

Therefore the principal part of $\left(\wp^{\prime}\right)^{-2}$ at $\omega_{j}$ is $\frac{1}{4 a_{j}^{2}\left(x-\omega_{j}\right)^{2}}$. According to (59), $a_{j}=$ $\frac{1}{2} \wp^{\prime \prime}\left(\omega_{j}\right)=3 e_{j}^{2}-\frac{g_{2}}{4}$. The principal part of $\wp(x)$ at the origin is $\frac{1}{x^{2}}$. Thus

$$
A_{j}=\frac{1}{\left(6 e_{j}^{2}-\frac{g_{2}}{2}\right)^{2}}=\left[\frac{1}{2\left(e_{j}-e_{i}\right)\left(e_{j}-e_{k}\right)}\right]^{2},
$$

where the indices $i, j, k$ are a permutation of $1,2,3$. The latter expression follows from

$$
\begin{gathered}
3 e_{j}^{2}-\frac{g_{2}}{4}=2 e_{j}^{2}+e_{j}\left(e_{j}+e_{i}\right)+e_{j} e_{k}+e_{i} e_{k}=2 e_{j}^{2}+e_{i} e_{k}=2\left(e_{i}+e_{k}\right)^{2}+e_{i} e_{k} \\
=\left(2 e_{i}+e_{k}\right)\left(2 e_{k}+e_{i}\right)=\left(e_{i}-e_{j}\right)\left(e_{k}-e_{j}\right),
\end{gathered}
$$

where (17) and (60) were taken into account.

Direct computations, based on (17) and (60), show that

$$
\sum_{j=1}^{3} e_{j}^{2}=\frac{g_{2}}{2}, \quad \sum_{i<k} e_{i}^{2} e_{k}^{2}=\frac{g_{2}^{2}}{16}, \quad \text { and } \quad \sum_{j=1}^{3} e_{j}^{3}=\frac{3}{4} g_{3},
$$

where summation in the second expression is taken over all possible pairs $i, k$ such that $1 \leq i<k \leq 3$. Then

$$
\sum_{j=1}^{3} A_{j}=\frac{\sum_{i<k}\left(e_{i}-e_{k}\right)^{2}}{4 \prod_{i<k}\left(e_{i}-e_{k}\right)^{2}}=\frac{3 g_{2}}{8 \prod_{i<k}\left(e_{i}-e_{k}\right)^{2}} .
$$

Next we compute the denominator, using (60) and (63):

$$
\begin{gathered}
-8 \prod_{i<k}\left(e_{i}-e_{k}\right)^{2}=\prod_{j=1}^{3}\left(6 e_{j}^{2}-\frac{g_{2}}{2}\right)=-\frac{g_{2}^{3}}{8}+6 \frac{g_{2}^{2}}{4} \sum_{j=1}^{3} e_{j}^{2}-18 g_{2} \sum_{i<k} e_{i}^{2} e_{k}^{2} \\
+6^{3} \prod_{j=1}^{3} e_{j}^{2}=\frac{1}{2}\left(27 g_{3}^{2}-g_{2}^{3}\right)=-\frac{\Delta}{2} .
\end{gathered}
$$

Copyright $@$ by SIAM. Unauthorized reproduction of this article is prohibited. 
Now the second statement of the lemma follows from (66).

In order to evaluate $A_{0}$ we put $z=0$ at (55). Since $\wp^{\prime}$ has a pole at the origin, (55) yields

$$
A_{0}=-\sum_{j=1}^{3} A_{j} e_{j}=\frac{\sum_{j=1}^{3} e_{j}\left(e_{i}-e_{k}\right)^{2}}{-4 \prod_{i<k}\left(e_{i}-e_{k}\right)^{2}}
$$

Using (17) and (65), we calculate the numerator in the latter expression as $-\frac{9}{4} g_{3}$. Then the first statement of the lemma follows from (67)-(68).

Now, the combination of (45)-(46) and of (58)-(59) yields

$$
v_{2}(z)=\frac{1}{6 \varepsilon^{4}}\left[\left[A_{0} x-A_{+} \zeta(x)\right] \wp^{\prime}(x)-2 \sum_{j=1}^{3} A_{j}\left(\wp(x)-e_{i}\right)\left(\wp(x)-e_{k}\right)\right],
$$

where for convenience we use the notation $x=\varepsilon z$. The latter sum is a quadratic polynomial in $\wp$, which, according to (17) and (68), can be written as $-2 A_{+} \wp^{2}+$ $2 A_{0} \wp+B$, where the constant $B=-2 \sum_{j=1}^{3} A_{j} e_{i} e_{k}$. In order to determine $B$, note that (46) implies that $v_{2}$ is an even function, which has zero of order four at the origin. Using the Laurent expansions of $\wp, \wp^{\prime}$, and $\zeta$ at the origin (see [GR]) and equating the constant term of (69) to be zero, we obtain

$$
B=-2 \sum_{j=1}^{3} A_{j} e_{i} e_{k}=A_{+}\left(\frac{g_{2}}{10}+\frac{g_{2}}{30}\right)+2 A_{+} \frac{g_{2}}{10}=\frac{2 g_{2}^{2}}{\Delta} .
$$

Combining the latter equation with (69) and Lemma 2.3 yields

$$
\begin{aligned}
v_{2}(z) & =\frac{1}{\varepsilon^{4} \Delta}\left(\left[\frac{3 g_{3}}{2} \varepsilon z-g_{2} \zeta(\varepsilon z)\right] \wp^{\prime}(\varepsilon z)-2 g_{2} \wp^{2}(\varepsilon z)+3 g_{3} \wp(\varepsilon z)+\frac{g_{2}^{2}}{3}\right) \\
& =\frac{1}{\varepsilon^{4} \Delta}\left(\frac{3 g_{3}}{2}\left[\varepsilon z \wp^{\prime}(\varepsilon z)+2 \wp(\varepsilon z)\right]-\frac{g_{2}}{3}\left[3 \zeta(\varepsilon z) \wp^{\prime}(\varepsilon z)+6 \wp^{2}(\varepsilon z)-g_{2}\right]\right) .
\end{aligned}
$$

Note that $v_{2}(z)$ is an elliptic function if and only if $\frac{3 g_{3}}{2} x-g_{2} \zeta(x)$ is doubly periodic with fundamental periods $2 \omega_{1}, 2 \omega_{3}$. Using $\zeta\left(x+2 \omega_{j}\right)=\zeta(x)+2 \zeta\left(\omega_{j}\right)$ for all $x$ in the domain of $\zeta$ and $j=1,2,3[\mathrm{GR}]$, we can write the condition that $v_{2}$ is an elliptic function as

$$
3 g_{3} \omega_{j}-2 g_{2} \zeta\left(\omega_{j}\right)=0, \quad j=1,3 .
$$

Considering these equations as a linear system for unknowns $3 g_{3},-2 g_{2}$, we come to the conclusion that it cannot have nontrivial solutions since its determinant $\omega_{1} \zeta\left(\omega_{3}\right)-$ $\omega_{3} \zeta\left(\omega_{1}\right) \equiv-\frac{i \pi}{2}[\mathrm{GR}]$. Thus $v_{2}$ is not an elliptic function for any energy $C \in\left(-\frac{1}{3}, 0\right)$. Note that in the case of the separatrix solution $v_{2}$ is also not a hyperbolic function (see $[\mathrm{To} 4])$.

2.4. Estimates for $\boldsymbol{v}_{\mathbf{1}}(\boldsymbol{z}), \boldsymbol{v}_{\mathbf{2}}(\boldsymbol{z})$. Let $T$ denote trapezoid $A B C D$ in $\mathbb{C}$ with the vertices $A\left(\tilde{\omega}_{1}-\tilde{\omega}_{3}\right), B\left(\tilde{\omega}_{1}+2 \tilde{\omega}_{3}\right), C\left(\frac{3}{2} \tilde{\omega}_{3}\right), D\left(-\frac{1}{2} \tilde{\omega}_{3}\right)$. We start with the estimate

$$
\left|q^{2}(z, \varepsilon)\right| \leq \frac{Q}{|z|^{2}}
$$

Copyright $@$ by SIAM. Unauthorized reproduction of this article is prohibited. 
with some $Q>0$, which is valid for any $\varepsilon \geq 0$ and for any $z$ in the trapezoid $T$. The value of the constant $Q$ can be chosen as $Q=1+\left|\omega_{2}\right|^{2} \tilde{Q}$, where $\tilde{Q}=$ $\frac{1}{12}+\max _{x \in \tilde{T}}\left|\wp(x)-\frac{1}{x^{2}}\right|$. Here $\tilde{T}$ denotes the trapezoid in the $x$-plane, $x=\varepsilon z$, that corresponds to $T$. Note that $\omega_{2}$ is defined by the energy $J(\varepsilon)$ of the periodic solution (33). However, we can choose $Q$ such that (73) holds for all $J(\varepsilon) \in E$.

Lemma 2.4. There exist positive constants $A$ and $B$ such that the estimates

$$
\left|v_{1}(z)\right| \leq \frac{A}{|z|^{3}} \quad \text { and } \quad\left|v_{2}(z)\right| \leq B|z|^{4}
$$

are valid in $T$ for any $\varepsilon \geq 0$ and any $J(\varepsilon) \in E$.

Proof. The first estimate follows from (45) and the inequality

$$
\left|\wp^{\prime}(x)+\frac{2}{x^{3}}\right| \leq A^{3}
$$

in $\tilde{T}$, similarly to $(73)$.

The second inequality (74) follows from the fact that the expression in the square brackets in the right-hand side of (69) is bounded by $6|x|^{4} B$ in $\tilde{T}$ with some positive constant $B$ that does not depend on $\varepsilon$ and on $J(\varepsilon) \in E$.

\section{Solution by iterations.}

3.1. Operator $\left(D^{2}+1\right)^{-1}$. Let $\omega \in \mathbb{C}, a>0$, and let an analytic function $g(z)$ be real-valued on $\Re z=\Re \omega$ and symmetrical with respect to $\omega$. Note that under these assumptions $g(z)$ is also real-valued along $\Im z=\Im \omega$.

Proposition 3.1. The operator $I_{a}^{\omega}$ defined by

$$
I_{a}^{\omega} g(z)=\frac{1}{2 i}\left[e^{i z} \int_{\omega-i a}^{z} e^{-i \xi} g(\xi) d \xi-e^{-i z} \int_{\omega+i a}^{z} e^{i \xi} g(\xi) d \xi\right]
$$

is inverse to $D^{2}+1$, and $I_{a}^{\omega} g(z)$ is real-valued on $\Re z=\Re \omega$ and symmetrical with respect to $\omega$.

Proof. The first statement of the proposition is obvious. In order to prove the second statement, we decompose

$$
I_{a}^{\omega} g(z)=I^{\omega} g(z)+\frac{1}{2 i}\left[e^{i z} \int_{\omega-i a}^{\omega} e^{-i \xi} g(\xi) d \xi-e^{-i z} \int_{\omega+i a}^{\omega} e^{i \xi} g(\xi) d \xi\right],
$$

where $I^{\omega}=I_{0}^{\omega}$. Let $i b=\int_{\omega}^{\omega+i a} e^{i(\xi-\omega)} g(\xi) d \xi$. Since $g$ is real-valued along the path of integration, $b \in \mathbb{R}$ and $-i b=\int_{\omega}^{\omega-i a} e^{-i(\xi-\omega)} g(\xi) d \xi$. Thus, the second term in (77) is symmetrical and real-valued as it is

$$
\frac{1}{2 i}\left[i b e^{i(z-\omega)}+i b e^{-i(z-\omega)}\right]=b \cos (z-\omega) .
$$

To complete the proof we observe that

$$
\begin{aligned}
I^{\omega} & =\frac{1}{2 i}\left[e^{i z} \int_{\omega}^{z} e^{-i \xi} g(\xi) d \xi-e^{-i z} \int_{\omega}^{z} e^{i \xi} g(\xi) d \xi\right]=\int_{\omega}^{z} \sin (z-\xi) g(\xi) d \xi \\
& =\sin (z-\omega) \int_{\omega}^{z} \cos (\xi-\omega) g(\xi) d \xi-\cos (z-\omega) \int_{\omega}^{z} \sin (\xi-\omega) g(\xi) d \xi
\end{aligned}
$$

Copyright $@$ by SIAM. Unauthorized reproduction of this article is prohibited. 
is also symmetrical with respect to $\omega$ and real-valued.

Using the reflection principle, it is easy to show that the function $g(z)$ of Proposition 3.1 is periodic with real or purely imaginary period $2 P$ if and only if it is symmetrical with respect to $\omega+P$. Then Proposition 3.1 shows that there is a (real) one-parameter family of operators $I_{a}^{\omega}$, preserving the symmetry of $g$ at $\omega$. The following proposition shows that symmetry can be preserved at both $\omega$ and $\omega+P$ if $P$ is not a multiple of $\pi$. If $P$ is a multiple of $\pi$, one can prove that a symmetrical and periodic $\left(D^{2}+1\right)^{-1} g(z)$ exists if and only if the Fourier expansion of $g(z)$ does not contain a $\cos z$ term.

Proposition 3.2. Let $g(z)$ be a function, symmetrical with respect to $\omega$ and $\omega+P$ and real-valued on $[\omega, \omega+P]$, where $P$ is either real or purely imaginary. If $P \neq k \pi, k \in \mathbb{N}$, then there exists an operator $I$ that is inverse to $D^{2}+1$ and such that Ig inherits the abovementioned properties of $g$.

Proof. To satisfy the requirements at $\omega$, the operator $I$ has to be of the form $I g=I^{\omega} g+d \cos (z-\omega)$, where $d \in \mathbb{R}$ (and depends on $g$ ). Then

$$
\begin{aligned}
& I g=I^{\omega+P} g+d \cos (z-\omega) \\
& +\frac{1}{2 i}\left[e^{i(z-\omega-P)} \int_{\omega}^{\omega+P} e^{-i(\xi-\omega-P)} g(\xi) d \xi-e^{-i(z-\omega-P)} \int_{\omega}^{\omega+P} e^{i(\xi-\omega-P)} g(\xi) d \xi\right] .
\end{aligned}
$$

Let $A_{1,2}$ denote the (two) terms in the square brackets, respectively, and let $A=$ $\frac{1}{2}\left(A_{1}+A_{2}\right), B=\frac{1}{2}\left(A_{1}-A_{2}\right)$. Then the square bracket expression in (80) becomes $A \sin (z-\omega-P)-i B \cos (z-\omega-P)$. Therefore

$$
I g=I^{\omega+P} g+(d \cos P-i B) \cos (z-\omega-P)+(A-d \sin P) \sin (z-\omega-P) .
$$

Using (79) we see that the first two terms in the right-hand side of (81) are symmetrical at $\omega+P$. Thus $I g$ is symmetrical with respect to $\omega+P$ if and only if

$$
d=\frac{2 \int_{\omega}^{\omega+P} \cos (\xi-\omega-P) g(\xi) d \xi}{\sin P} .
$$

Equation (82) can be satisfied for any $P \neq k \pi, k \in \mathbb{Z}$. Noting that $d$ and $i B$ are real for both real and purely imaginary $P$ concludes the proof.

3.2. Estimates for the operator $\mathcal{I}_{2}$. Consider the trapezoid $T$ introduced in section 2.4. Given a number $z_{1} \in\left(0, \frac{1}{4} \tilde{\omega}_{1}\right)$, which is independent of $\varepsilon$, we cut a triangle near the origin with vertices $z_{1}, z_{2}, z_{3}$, where $z_{2}, z_{3}$ are the points on the intersection of the imaginary axis and the lines through the points $z_{1}, A$ and the points $z_{1}, B$, respectively. The obtained region is denoted by $T_{z_{1}}$. Clearly, for a given $z_{0}>0$ we have $R_{z_{0}} \subset T_{z_{1}}$ if $z_{0}$ is large enough (i.e., according to (52), if $\varepsilon_{0}$ is small enough). We define the operator

$$
\mathcal{I}_{2}=I_{-2 i \tilde{\omega}_{3}}^{\tilde{\omega}_{2}} .
$$

For a given $z \in T_{z_{1}}, \gamma_{ \pm}(z)$ denote contours of integration $[z, B]$ and $[z, A]$, respectively; see Figure 5.

Proposition 3.3. The inequality

$$
\left|\frac{\xi}{z}\right|>\frac{\omega_{1}}{\sqrt{\omega_{1}^{2}-\frac{25}{4} \omega_{3}^{2}}}
$$

Copyright (C) by SIAM. Unauthorized reproduction of this article is prohibited. 


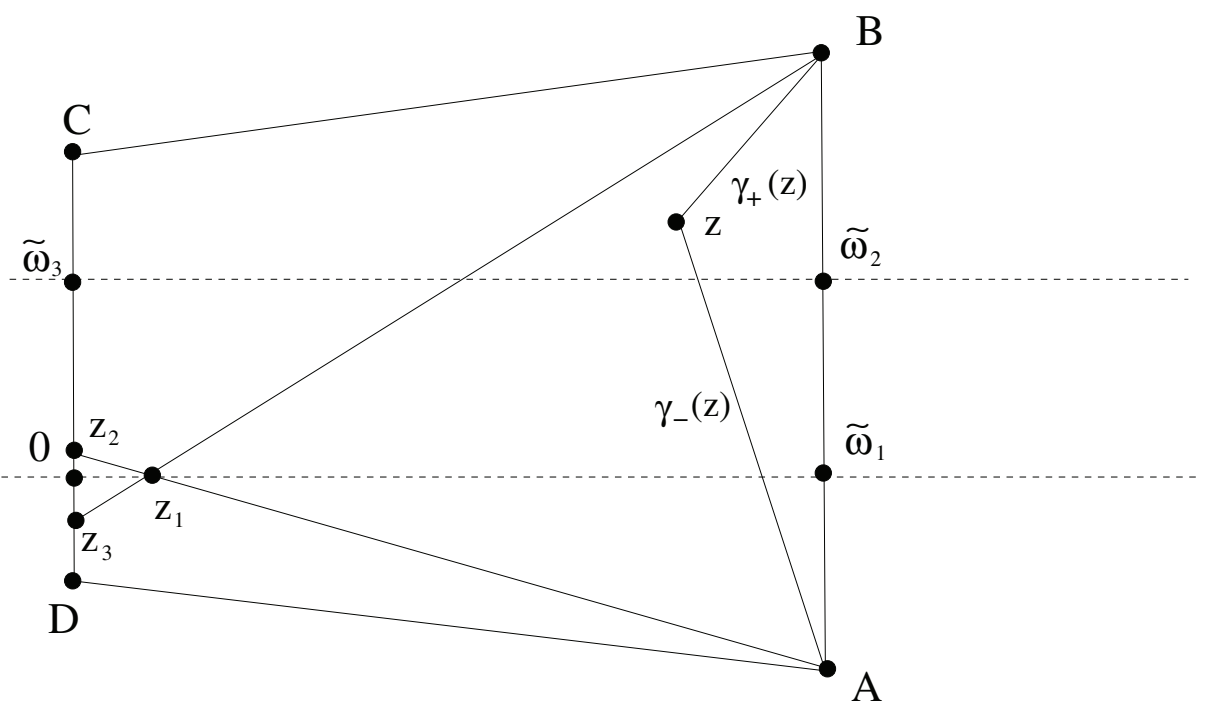

FIG. 5. Region $T_{z_{1}}$ and contours $\gamma_{ \pm}(z)$.

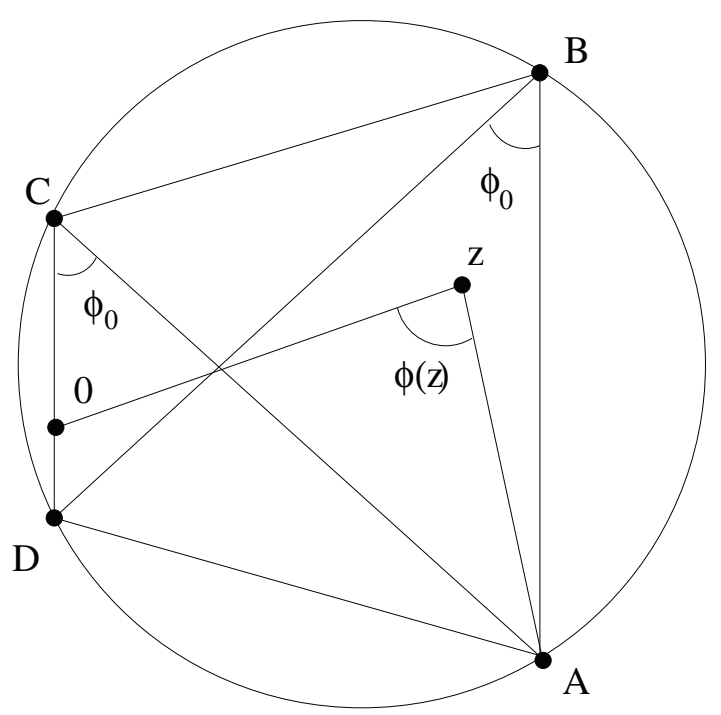

FIG. 6. Trapezoid $A B C D$ and angles $\phi(z), \phi_{0}$.

holds for all $z \in T_{z_{1}}$ and all $\xi \in \gamma_{+}(z) \cap \gamma_{-}(z)$.

Proof. For an arbitrary $z \in T_{z_{1}}$, let $\phi(z)$ denote the angle between the segments $\gamma_{-}(z)$ and $[0, z]$ (see Figure 6), and let $\alpha(z)=\min _{\xi \in \gamma_{-}(z)} \mid \frac{|\xi|}{|z|}$. We choose the acute angle for $\phi\left(\tilde{\omega}_{1}+2 \tilde{\omega}_{3}\right)$ (at the vertex $B$ ) and then define $\phi(z)$ in $T_{z_{1}}$ by continuity. Clearly $\alpha(z)=1$ if $\phi(z) \geq \frac{\pi}{2}$ and $\alpha(z)=\sin \phi(z)$ if $\phi(z)<\frac{\pi}{2}$. The latter case can happen only if $\Im z \geq 0$; hence $\alpha(z)=1$ if $\Im z<0$. Let $\phi_{0}$ be the acute angle between $A B$ and $D B$. We show that $\phi(z) \geq \phi_{0}$ for every $z \in T_{z_{1}}$.

Indeed, let us inscribe the right trapezoid $A B C D$ into a circle. Then (at the vertex $C) \phi\left(\frac{3}{2} \tilde{\omega}_{3}\right)=\phi_{0}$. Simple geometrical considerations (see Figure 6 ) imply that

Copyright (c) by SIAM. Unauthorized reproduction of this article is prohibited. 
$\min _{z \in T_{z_{1}}} \phi(z)$ is attained at $z=\frac{3}{2} \tilde{\omega}_{3}$. Thus, $\alpha(z) \geq \sin \phi_{0}$. Therefore

$$
\alpha(z) \geq \sin \phi_{0}=\frac{\omega_{1}}{\sqrt{\omega_{1}^{2}-\frac{25}{4} \omega_{3}^{2}}}
$$

for any $z \in T_{z_{1}}$. The same estimate holds for the contour $\gamma_{+}(z)$. The proof is completed.

Proposition 3.4. For any $z \in T_{z_{1}}$

$$
\left|\mathcal{I}_{2} f(z)\right| \leq \sqrt{1-4 \frac{\omega_{1}^{2}}{\omega_{3}^{2}}} \max _{\xi \in \gamma_{+}(z) \cup \gamma_{-}(z)}|f(\xi)|,
$$

where $f$ is a continuous function on $T_{z_{1}}$.

Proof. According to the construction of $\gamma_{+}, \gamma_{-}$,

$$
\Re[i(\xi-z)] \leq-\frac{\left|\omega_{3}\right|}{\sqrt{4 \omega_{1}^{2}-\omega_{3}^{2}}}|z-\xi| \quad \text { and } \quad \Re[i(z-\xi)] \leq-\frac{\left|\omega_{3}\right|}{\sqrt{4 \omega_{1}^{2}-\omega_{3}^{2}}}|z-\xi|
$$

for all $\xi \in \gamma_{ \pm}(z)$, respectively. Therefore

$$
\begin{aligned}
\left|\mathcal{I}_{2} f(z)\right| & \leq \frac{1}{2}\left|\int_{\gamma_{-}(z)} e^{i(z-\xi)} f(\xi) d \xi+\int_{\gamma_{-}(z)} e^{i(z-\xi)} f(\xi) d \xi\right| \\
& \leq \max _{\xi \in \gamma_{+}(z) \cup \gamma_{-}(z)}|f(\xi)| \int_{0}^{\infty} e^{-\left(\left|\omega_{3}\right| / \sqrt{4 \omega_{1}^{2}-\omega_{3}^{2}}\right) \lambda} d \lambda
\end{aligned}
$$

which implies (86).

LEMMA 3.5. If

$$
|f(z)| \leq \frac{1}{|z|^{a}}
$$

in $T_{z_{1}}$ with $a \geq 0$, then

$$
\left|\mathcal{I}_{2} f(z)\right| \leq \frac{\beta}{|z|^{a}}
$$

where $\beta=\left(1-\frac{25 \omega_{3}^{2}}{4 \omega_{1}^{2}}\right)^{\frac{1}{2}}\left(1-4 \frac{\omega_{1}^{2}}{\omega_{3}^{2}}\right)^{\frac{a}{2}}$.

This lemma is a direct consequence of Propositions 3.3 and 3.4.

3.3. Estimates for the operator $\mathcal{I}_{1}$. Solution $\mathcal{I}_{1} g$ to linear differential equation (43), where $g$ denotes the right-hand side of (43), that we consider in the paper is given by $(50)$. In order to estimate $\mathcal{I}_{1}$, we need the following simple statement.

Proposition 3.6. Let a ray $\lambda$ on the complex $z$-plane lie outside the disk $|z|<a$, $a>0$. Then for any $\alpha \geq 2$

$$
\left|\int_{\lambda} \frac{d z}{z^{\alpha}}\right| \leq \frac{\pi}{a^{\alpha-1}}
$$

Proof. Let $m$ denote the line containing the ray $\lambda$. Suppose $m$ is tangent to the disk $|z|<a$ at the point $\omega$. If $z \in m$, then $|z|^{2}=a^{2}+x^{2}$, where $x=|z-\omega|$. Then

$$
\left|\int_{\lambda} \frac{d z}{z^{\alpha}}\right| \leq \int_{-\infty}^{\infty} \frac{d x}{\left(a^{2}+x^{2}\right)^{\frac{\alpha}{2}}} \leq \frac{2}{a^{\alpha-2}} \int_{0}^{\infty} \frac{d x}{\left(a^{2}+x^{2}\right)}=\frac{\pi}{a^{\alpha-1}}
$$

Copyright (c) by SIAM. Unauthorized reproduction of this article is prohibited. 
This estimate will definitely hold if $m$ does not intersect the closed disk $|z| \leq a$.

Suppose now that $m$ intersects the disk $|z|<a$. Let $\omega$ denote the vertex of the ray $\lambda$, and let $\zeta$ denote one of the two points which lie on the distance $a$ from both $\omega$ and $m$. Then $|\xi-\zeta|<|\xi|$ for any $\xi \in \lambda$, and we can apply the estimate (92) again, placing the origin at $\zeta$.

Notice that estimates of Proposition 3.3 are applicable to the contours in (50). Then, using Lemma 2.4 and Proposition 3.6, we can easily establish the following estimates.

Lemma 3.7. If $|g(z)| \leq K|z|^{-n}, n \geq 6$, or if $|g(z)| \leq K|z|^{-4}$ in $T_{z_{1}}$, then

$$
\left|\mathcal{I}_{1} g(z)\right| \leq \frac{M_{1} K}{\left|z^{n-2}\right|} \quad \text { and } \quad\left|\mathcal{I}_{1} g(z)\right| \leq \frac{M_{2} K}{\varepsilon\left|z^{-3}\right|}
$$

respectively, where $M_{1}=\left(1-\frac{25}{4} \frac{\omega_{3}^{2}}{\omega_{1}^{2}}\right)^{\frac{n-5}{2}} \pi A B$ and $M_{2}=\max \left\{2\left|\omega_{3}\right|, \sqrt{\omega_{1}^{2}-\frac{9}{4} \omega_{3}^{2}}\right\} A B$.

3.4. Proof of Theorem 2.1. Proof. We start with the obvious observation that

$$
\varepsilon \leq \frac{\sqrt{\omega_{1}^{2}-4 \omega_{3}^{2}}}{|z|}
$$

if $z \in T_{z_{1}}$. Using the first inequality in (53), we obtain that $f(q)$ from (36) satisfies

$$
|f| \leq \tilde{K}_{0}|z|^{-6}+\varepsilon \tilde{K}_{1}|z|^{-4}
$$

in $T_{z_{1}}$ with some positive constants $\tilde{K}_{0}, \tilde{K}_{1}$ that do not depend on $\varepsilon$ and $J(\varepsilon) \in E$. Thus, according to (49) and the estimates for $\mathcal{I}_{1,2}$,

$$
\left|\mathcal{N} w_{0}\right| \leq \frac{K}{2}\left(\frac{\varepsilon^{\alpha-1}}{|z|^{3}}+\frac{1}{|z|^{4}}\right)
$$

in $T_{z_{1}}$ for some constant $K>0$ that does not depend on $\varepsilon, J(\varepsilon)$, and $z_{1}$.

Let us now prove by induction that by choosing a sufficiently large $z_{1}>0$ we can find $\delta>0$, such that the estimate

$$
\Delta w_{n} \leq(\delta M)^{n-1} \frac{K}{|z|^{3}}, \quad \text { where } \quad \delta M \leq \frac{1}{2},
$$

holds in $T_{z_{1}}$ for all $n=1,2, \ldots$, all $J(\varepsilon) \in E$, and all sufficiently small $\varepsilon$. Here $M=\max \left\{M_{1}, M_{2}\right\}$, where $M_{1}$ is given in Lemma 3.7 with $n=6$.

According to Lemma 2.4, the estimate for $v_{22}$ for all $z \in T_{z_{1}}$ and $J(\varepsilon) \in E$ is

$$
\left|v_{22}(z)\right| \leq B|z|^{4}+\frac{L}{\varepsilon^{7}|z|^{3}}
$$

for some $L>0$. Then, according to (94), (96), and (53), we can choose $K>0$ to be so large that $w_{1}=\mathcal{N} w_{0}+\varepsilon^{6} b(\varepsilon) v_{22}$ satisfies

$$
\left|w_{1}\right| \leq \frac{K}{|z|^{3}}
$$

in $T_{z_{1}}$ for all $J(\varepsilon) \in E$ and all sufficiently small $\varepsilon$. Thus, for $n=1,(97)$ has been established. Assume that this estimate is true for $k=1,2, \ldots, n$, and let us establish it for $k=n+1$. First, we can represent

$$
\begin{aligned}
\Delta w_{n+1}= & \mathcal{I}_{1} \circ\left[\left(1-12 c q^{2} \mathcal{I}_{2}\right)\left[\mathcal{I}_{2}\left(w_{n}+w_{n-1}\right) \cdot \mathcal{I}_{2} \Delta w_{n}\right]-12 q^{2}(1-c) \Delta w_{n}\right. \\
& \left.+12 c q^{2} \mathcal{I}_{2}\left(\varepsilon^{2}+12 c q^{2} \mathcal{I}_{2}\right) \Delta w_{n}\right] .
\end{aligned}
$$

Copyright $@$ by SIAM. Unauthorized reproduction of this article is prohibited. 
Taking into account (99) and Lemma 3.5, we obtain the estimates

(101)

$\frac{12 Q l \varepsilon^{\alpha}}{|z|}\left|\frac{\Delta w_{n}}{z}\right|, 12 c Q \beta\left[\frac{\varepsilon}{|z|}\left|\frac{\varepsilon \Delta w_{n}}{z}\right|+\frac{12 c Q \beta}{|z|}\left|\frac{\Delta w_{n}}{z^{3}}\right|\right], 4 K \beta^{2}\left(1+\frac{12 c Q \beta}{|z|^{2}}\right)\left|\frac{\Delta w_{n}}{z^{3}}\right|$

for the second, third, and first (nonlinear) terms in the square brackets in (100), respectively. For the latter estimate, observe that both $w_{n}$ and $w_{n-1}$ are bounded by $\frac{2 K}{|z|^{3}}$ according to (97). (Note that the constants $\beta, K, M$ are independent of $z_{1}$.) Applying operator $\mathcal{I}_{1}$ to these three terms and utilizing Lemma 3.7, we obtain the estimates

$\frac{12 Q l}{|z|} \varepsilon^{\alpha-1} M\left|\Delta w_{n}\right|, 12 c Q \beta\left[\varepsilon+\frac{12 c Q \beta}{|z|}\right] \frac{M}{|z|}\left|\Delta w_{n}\right|, 4 K \beta^{2}\left(1+\frac{12 c Q \beta}{|z|^{2}}\right)\left|\frac{M \Delta w_{n}}{z}\right|$.

Factoring $M\left|\Delta w_{n}\right|$, we obtain $\left|\Delta w_{n+1}\right| \leq \delta M\left|\Delta w_{n}\right|$, where

$$
\delta=\frac{12 Q l \varepsilon^{\alpha-1}+12 c Q \beta \varepsilon}{|z|}+\frac{12^{2} c^{2} Q^{2} \beta^{2}}{|z|^{2}}+\frac{4 K \beta^{2}}{|z|}\left(1+\frac{12 c Q \beta}{|z|^{2}}\right) .
$$

By choosing a sufficiently large $z_{1}>0$ and sufficiently small $\varepsilon_{0}>0$, we can guarantee the condition $\delta M \leq \frac{1}{2}$ in $T_{z_{1}}$ for all $\varepsilon \leq \varepsilon_{0}$ and for all $J(\varepsilon) \in E$. Thus, (97) holds for $k=n+1$. We can choose some $z_{0} \geq z_{1}$ so that $R_{z_{0}} \subset T_{z_{1}}$ and $\varepsilon_{0} z_{0} \leq \max \left\{\omega_{1},\left|\omega_{3}\right|\right\}$ (taking smaller $\varepsilon_{0}$ if necessary). Thus, inequalities (97) prove convergence of iterations to a solution of (42) that is uniform in $R_{z_{0}} \times\left(0, \varepsilon_{0}\right]$ for all $J(\varepsilon) \in E$.

In the case $\varepsilon=0$ the region $T_{z_{1}}$ becomes the right half-plane with the appropriate cut, $q^{2}=\frac{6}{z^{2}}, f(q)=-\frac{6 !}{z^{6}}$, and the fundamental solutions $v_{1,2}$ to the homogeneous equations (43) become $v_{1}(z)=\frac{-12}{z^{3}}$ and $v_{2}(z)=\frac{-z^{4}}{84}$. Thus, for the case $\varepsilon=0$ Lemma 2.4 becomes trivial, and the proof of convergence of iterations $w=\sum_{1}^{\infty} \Delta w_{n}$ that solve (42) holds for the case $\varepsilon=0$. (Note that in this case the contours of integration in the integral operators $\mathcal{I}_{1,2}$ are the rays, emanating from $z$, with the slope $\frac{\Im w}{\Re w}$, where $w$ is the beginning of the corresponding contour in the case $\varepsilon>0$; in other words, $\arg w$ does not depend on $\varepsilon$.) Thus, the proof of Theorem 2.1 is completed.

Remark 3.8. It is easy to verify that $\alpha>1$ in Theorem 2.1 implies that there exist some $L_{n}>0$ such that for all $n \in \mathbb{N}$

$$
\left|\Delta w_{n}\right| \leq L_{n}|z|^{-n(1+\tilde{\alpha})-2}
$$

in $R_{z_{0}}$, where $\tilde{\alpha}=\min \{1, \alpha-1\}$. Moreover, for all $n \in \mathbb{N}$ there exist some $K_{n}>0$ such that

$$
\left|\sum_{j=n}^{\infty} \Delta w_{n}\right| \leq K_{n}|z|^{-n(1+\tilde{\alpha})-2} .
$$

Let $P \subset T$ denote the triangle with vertices $\tilde{\omega}_{3}, \tilde{\omega}_{1}$, and $\tilde{\omega}_{1}+2 \tilde{\omega}_{3}$. It is clear that $z \in P$ implies that all the contours of integration in operators $\mathcal{I}_{1,2}$ are in $P$. Notice that in $P$

$$
\min \left\{\omega_{1},\left|\omega_{3}\right|\right\} \leq \varepsilon|z| \leq \sqrt{\omega_{1}^{2}-4 \omega_{3}^{2}}
$$


The latter condition allows us to prove convergence of iterations in $P$ without the requirement (53) in Theorem 2.1.

COROllary 3.9. If instead of (53) in Theorem 2.1 we require only

$$
\lim _{\varepsilon \rightarrow 0} b(\varepsilon)=\lim _{\varepsilon \rightarrow 0}[c(\varepsilon)-1]=0,
$$

the statement of Theorem 2.1 is still valid in $P \times\left[0, \varepsilon_{0}\right]$; i.e., there exists some $\varepsilon_{0}>0$ such that the series (54) is uniformly convergent in $P \times\left[0, \varepsilon_{0}\right]$.

Proof. The function

$$
h(\varepsilon)=\max \{|b(\varepsilon)|,|c(\varepsilon)-1|\}
$$

is continuous and nonnegative, and $\lim _{\varepsilon \rightarrow 0} h(\varepsilon)=0$. Then inequalities (95) and (96) become

$$
|f| \leq \tilde{K}_{0}|z|^{-6}+h(\varepsilon) \tilde{K}_{1}|z|^{-4} \quad \text { and } \quad\left|\mathcal{N} w_{0}\right| \leq \frac{K}{2}\left(\frac{h(\varepsilon)}{\varepsilon|z|^{3}}+\frac{1}{|z|^{4}}\right),
$$

respectively. Then, as in Theorem 2.1, we can choose $K>0$ so large that

$$
\left|w_{1}\right| \leq \frac{\tilde{h}(\varepsilon) K}{|z|^{2}}
$$

in $P$, where $\tilde{h}(\varepsilon)=\max \left\{h(\varepsilon), \varepsilon^{2}\right\}$.

As in Theorem 2.1, we will prove convergence of (54) by induction if we show that by choosing a sufficiently small $\varepsilon_{0}>0$ we can find $\delta>0$, such that the estimate

$$
\Delta w_{n} \leq(\delta M)^{n-1} \frac{\tilde{h}(\varepsilon) K}{|z|^{2}}, \quad \text { where } \quad \delta M \leq \frac{1}{2},
$$

holds in $P$ for all $n=1,2, \ldots$ and all $J(\varepsilon) \in E$. Taking into account (111) and Lemma 3.5, we obtain the estimates

$$
\begin{gathered}
12 Q l \tilde{h}(\varepsilon)\left|\frac{\Delta w_{n}}{z^{2}}\right|, \\
12 c Q \beta\left[\left|\frac{\varepsilon^{2} \Delta w_{n}}{z^{2}}\right|+\frac{12 c Q \beta}{|z|^{2}}\left|\frac{\Delta w_{n}}{z^{2}}\right|\right], \quad 4 \tilde{h}(\varepsilon) K \beta^{2}\left(1+\frac{12 c Q \beta}{|z|^{2}}\right)\left|\frac{\Delta w_{n}}{z^{2}}\right|
\end{gathered}
$$

for the second, third, and first (nonlinear) terms in the square brackets in (100), respectively. Making sure that $\hat{h}(\varepsilon)$ and $\left|z^{-2}\right|$ are sufficiently small by choosing a sufficiently small $\varepsilon_{0}$ and using Lemma 3.7 , we can repeat the arguments of Theorem 2.1 in order to complete the proof of convergence of iterations (54).

Remark 3.10. In fact, we can prove convergence of iterations (54) in larger domains than those stated in Theorem 2.1 and Corollary 3.9. Fix some $n \in \mathbb{N}$. For Theorem 2.1, we replace the trapezoid $T$ with vertices $A B C D$ by the (concave) hexagon $\mathcal{T}$ with vertices $A O N B C D$, where $O=\tilde{\omega}_{1}, N=n \tilde{\omega}_{1}+\tilde{\omega}_{3}$, and other vertices are the same as in $T$; see Figure 7 . For $z \in \mathcal{T}$, contours of integration in operators $\mathcal{I}_{1,2}$ are the same as before, except for contour $\gamma_{-}(z)$ that is now the union of segments $[z, O]$ and $[O A]$. For Corollary 3.9, we replace the triangle $P$ with vertices $\tilde{\omega}_{3}, \tilde{\omega}_{1}$, $\tilde{\omega}_{1}+2 \tilde{\omega}_{3}$ by a quadrilateral $\mathcal{P}$ with vertices $\tilde{\omega}_{1}, n \tilde{\omega}_{1}+\tilde{\omega}_{3}, \tilde{\omega}_{1}+2 \tilde{\omega}_{3}, \tilde{\omega}_{3}$. For $z \in \mathcal{P}$, contours of integration in operators $\mathcal{I}_{1,2}$ are the same as before. It is easy to verify that the estimates for operators $\mathcal{I}_{1,2}$ from Lemmas 3.7 and 3.5 hold in domains $\mathcal{T}$ and $\mathcal{P}$, possibly with larger constants that depend on $n$. Now proofs of Theorem 2.1 and Corollary 3.9 can be extended to the domains $\mathcal{T}$ and $\mathcal{P}$, respectively. 


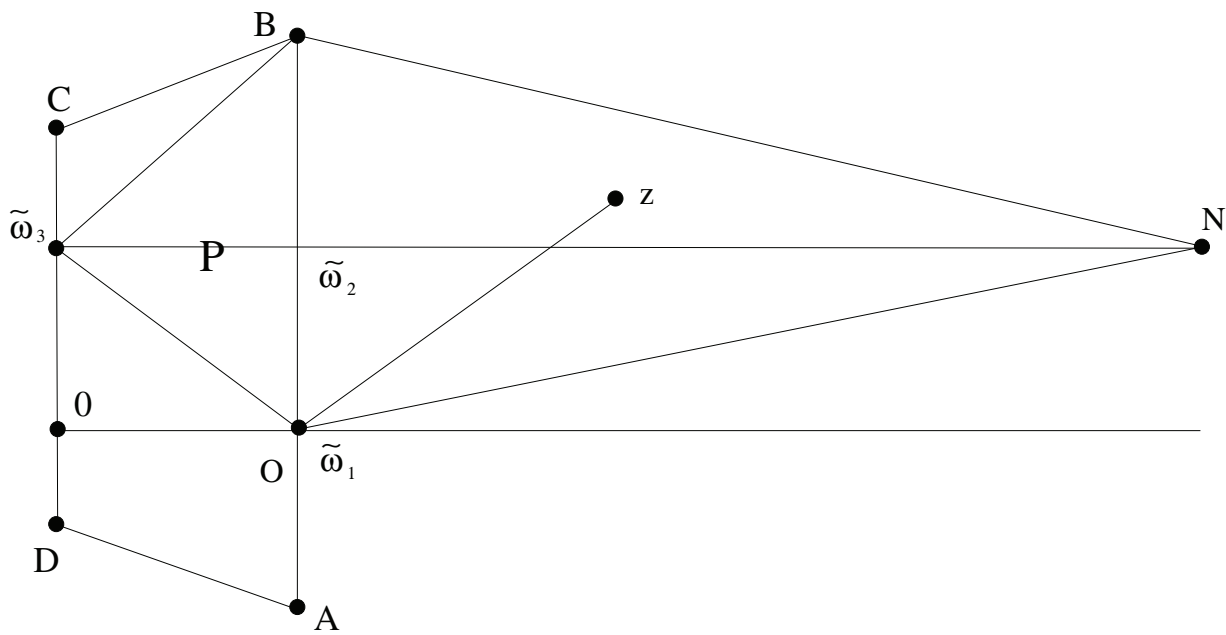

FIG. 7. Hexagon $\mathcal{T}$ with vertices $A O N B C D$. The contour $\gamma_{-}(z)$ is the union of segments $[z, O]$ and $[O A]$.

3.5. Symmetry at $\tilde{\boldsymbol{\omega}}_{\mathbf{2}}$. Here we will show that any solution to inner equation (23), obtained through iterations (54), is symmetrical with respect to $z=\tilde{\omega}_{2}$. Consequently, corresponding solutions to the outer equation (1), connected with (23) via (22), are symmetric at $x=\omega_{2}$.

The operator $\mathcal{I}_{1}$ can be represented in the following form.

Proposition 3.11. If a function $g(z)$ is analytic and bounded in $T_{z_{1}}$, then

$$
\mathcal{I}_{1} g=v_{1}(z) \int_{\tilde{\omega}_{2}}^{z} \frac{\int_{\tilde{\omega}_{2}}^{t} v_{1}(\tau) g(\tau) d \tau}{v_{1}^{2}(t)} d t
$$

Proof. Using (46) and integration by parts, one gets

$$
\begin{aligned}
\mathcal{I}_{1} g=-v_{1}(z) \int_{\tilde{\omega}_{2}}^{z}\left(v_{1}(t) g(t) \int_{0}^{t} \frac{d \tau}{v_{1}^{2}(\tau)}\right) d t+v_{2}(z) \int_{\tilde{\omega}_{2}}^{z} v_{1}(t) g(t) d t \\
=v_{1}(z) \times\left[-\left.\int_{\tilde{\omega}_{2}}^{t} v_{1}(\tau) g(\tau) d \tau \cdot \int_{0}^{t} \frac{d \tau}{v_{1}^{2}(\tau)}\right|_{\tilde{\omega}_{2}} ^{z}\right. \\
\left.+\int_{\tilde{\omega}_{2}}^{z} \frac{\int_{\tilde{\omega}_{2}}^{t} v_{1}(\tau) g(\tau) d \tau}{v_{1}^{2}(\tau)} d t+\int_{0}^{z} \frac{d t}{v_{1}^{2}(\tau)} \cdot \int_{\gamma(z)} v_{1}(t) g(t) d t\right] \\
=v_{1}(z) \lim _{\zeta \rightarrow \tilde{\omega}_{2}}\left[\int_{\zeta}^{z} v_{1}(t) g(t) d t \cdot \int_{0}^{\zeta} \frac{d t}{v_{1}^{2}(\tau)}\right]+v_{1}(z) \int_{\tilde{\omega}_{2}}^{z} \frac{\int_{\tilde{\omega}_{2}}^{t} v_{1}(\tau) g(\tau) d \tau}{v_{1}^{2}(\tau)} d t
\end{aligned}
$$

Note that the limit in the latter expression is zero because $\int_{\gamma(\zeta)} v_{1}(t) g(t) d t$ has a zero of order two at $\tilde{\omega}_{2}$, whereas $\int_{0}^{\zeta} \frac{d t}{v_{1}^{2}(\tau)}$ has a simple pole there.

Based on (45) and (73), solutions to the homogeneous equation (44) preserving the symmetry at the points $\tilde{\omega}_{j}, j=1,2,3$, are given by

$$
v_{2 j}(z)=v_{2}(z)-\frac{1}{6 \varepsilon^{7} \Delta}\left[\frac{3 g_{3}}{2} \omega_{j}-g_{2} \zeta\left(\omega_{j}\right)\right] v_{1}(z), \quad j=1,2,3,
$$

Copyright (C) by SIAM. Unauthorized reproduction of this article is prohibited. 
respectively. Indeed, direct calculations show that $D_{z} v_{2 j}\left(\tilde{\omega}_{j}\right)=0$. Then, according to $(44)$,

$$
D_{z}^{3} v_{2 j}=\left(\varepsilon^{2}+12 q^{2}\right) D_{z} v_{2 j}+12\left(q^{2}\right)^{\prime} v_{2 j}=0
$$

at $z=\tilde{\omega}_{j}$. By continuing this argument for higher derivatives, we prove symmetry of $v_{2 j}$ at $z=\tilde{\omega}_{j}$. Also, note that $v_{2 j}\left(\tilde{\omega}_{j}\right) \in \mathbb{R}$. Then, according to the differential equation for $v_{2 j}$, all even derivatives of $v_{2 j}$ at $z=\tilde{\omega}_{j}$ are also real.

The fact that operator $\mathcal{I}_{1}$ preserves the symmetry at the point $\tilde{\omega}_{2}$ and realvaluedness along the line $\Im z=\Im \tilde{\omega}_{2}$ is a direct consequence of Proposition 3.11. Thus, according to (36), $\Delta w_{1}$ is symmetric with respect to $\tilde{\omega}_{2}$ and real-valued on the line $\Im z=\Im \tilde{\omega}_{2}$. Then, according to (100) and to Proposition 3.1, the same is true for $w=\sum_{1}^{\infty} \Delta w_{n}$. Now, according to (34), the corresponding solution $v(z, \varepsilon)$ to $(23)$ satisfies

$$
v^{\prime}\left(\tilde{\omega}_{2}, \varepsilon\right)=v^{\prime \prime \prime}\left(\tilde{\omega}_{2}, \varepsilon\right)=0
$$

and is real-valued on $\Im z=\Im \tilde{\omega}_{2}$.

Thus, in Theorem 2.1 and Corollary 3.9 we have constructed a two-parameter family of solutions to (23), where $b(\varepsilon)$ and $c(\varepsilon)$ are the parameters, that are real along $\Im z=\Im \tilde{\omega}_{2}$ and satisfy (117).

3.6. Calculation of $\mathcal{I}_{1} \boldsymbol{f}(\boldsymbol{q})$. In order to calculate $\mathcal{I}_{1} f(q)$, we use (36) and (45) to evaluate the integral

$$
\begin{aligned}
\int_{\tilde{\omega}_{2}}^{t} v_{1}(\tau) f(q(\tau)) d \tau & =\int f(q) d\left(6 q^{2}\right) \\
& =\left.c\left[\frac{c-1-4 \varepsilon^{2}}{3}\left(6 q^{2}\right)^{3}-5 / 6\left(6 q^{2}\right)^{4}-2 \varepsilon^{6} C(\varepsilon)\left(6 q^{2}\right)\right]\right|_{\tilde{\omega}_{2}} ^{z}
\end{aligned}
$$

in (113). Switching to the original variable $x=\varepsilon z$ and using (113), we obtain

$$
\begin{aligned}
& \mathcal{I}_{1} f=6 \varepsilon^{3} c \wp^{\prime}(x) \\
& \times \int_{\omega_{2}}^{x} \frac{\left.\left(6 \wp(t)-\frac{1}{2}\right)\left[\frac{c-1-4 m^{2}}{3 \varepsilon}\left(6 \wp(s)-\frac{1}{2}\right)^{2}-\frac{5 \varepsilon}{6}\left(6 \wp(s)-\frac{1}{2}\right)^{3}-2 \varepsilon C(\varepsilon)\right]\right|_{s=\omega_{2}} ^{s=t} d t}{144 \prod_{1}^{3}\left(\wp(t)-e_{j}\right)}
\end{aligned}
$$

The integrand in (119) is nonsingular at $t=\omega_{2}$ since the numerator has zero at this point. Using this fact, we can cancel the factor $\left(\wp-e_{2}\right)$ in both the numerator and denominator. Then direct calculations show that the integrand in (119) becomes

$$
\begin{aligned}
\frac{c-1-4 \varepsilon^{2}}{3 \varepsilon} & \cdot \frac{\wp^{2}+\left(e_{2}-\frac{1}{4}\right) \wp+e_{2}^{2}-\frac{1}{4} e_{2}+\frac{1}{48}}{4\left(\wp-e_{1}\right)\left(\wp-e_{3}\right)} \\
- & \frac{\frac{5 \varepsilon}{6}\left(6 \wp(t)-\frac{1}{2}\right)^{4}}{144 \prod_{1}^{3}\left(\wp(t)-e_{j}\right)}-\frac{2 \varepsilon C(\varepsilon)\left(6 \wp(t)-\frac{1}{2}\right)}{144 \prod_{1}^{3}\left(\wp(t)-e_{j}\right)} .
\end{aligned}
$$

Copyright (c) by SIAM. Unauthorized reproduction of this article is prohibited. 
Let us focus on the first term of (120). Separating the integer part, we can rewrite it as

$$
\begin{aligned}
& \frac{c-1-4 \varepsilon^{2}}{3 \cdot 4 \varepsilon}\left[1-\frac{1}{4} \frac{\wp+e_{2}-\frac{1}{6}}{\left(\wp-e_{1}\right)\left(\wp-e_{3}\right)}\right] \\
& \quad=\frac{c-1-4 \varepsilon^{2}}{3 \cdot 4 \varepsilon}\left[1-\frac{B_{0}+B_{1} \wp\left(t-\omega_{1}\right)+B_{3} \wp\left(t-\omega_{3}\right)}{4}\right],
\end{aligned}
$$

where

$$
B_{1}=-\frac{e_{3}+\frac{1}{6}}{\left(e_{1}-e_{3}\right)^{2}\left(e_{1}-e_{2}\right)}, \quad B_{3}=\frac{e_{1}+\frac{1}{6}}{\left(e_{1}-e_{3}\right)^{2}\left(e_{2}-e_{3}\right)}, \quad \text { and } \quad B_{0}=-B_{1} e_{1}-B_{3} e_{3} .
$$

The expressions for $B_{1}, B_{3}$, and $B_{0}$ were obtained by evaluating the principal parts of the left-hand side of (122) at $t=\omega_{1}, t=\omega_{3}$, and $t=0$, respectively.

Using similar calculations, we obtain expressions

$$
\begin{aligned}
& -\frac{15 \varepsilon}{2} \wp(t)+\frac{5 \varepsilon}{2}-\frac{5 \varepsilon}{24}\left[E_{0}+E_{1 \wp}\left(t-\omega_{1}\right)+E_{3} \wp\left(t-\omega_{3}\right)\right] \quad \text { and } \\
& -\frac{\varepsilon C(\varepsilon)}{12}\left[D_{0}+D_{1 \wp}\left(t-\omega_{1}\right)+D_{3} \wp\left(t-\omega_{3}\right)\right]
\end{aligned}
$$

for the second and the third terms of (120), respectively, where

$$
D_{1}=\frac{1}{\left(e_{1}-e_{3}\right)^{2}\left(e_{1}-e_{2}\right)}, \quad D_{3}=-\frac{1}{\left(e_{1}-e_{3}\right)^{2}\left(e_{2}-e_{3}\right)}, \quad D_{0}=-D_{1} e_{1}-D_{3} e_{3}
$$

and

$$
E_{3}=-\frac{\frac{9}{4} e_{3}+\frac{3}{2} e_{2}+36 e_{2}^{3}-\frac{1}{3}}{\left(e_{1}-e_{3}\right)^{2}\left(e_{2}-e_{3}\right)}, \quad E_{0}=-E_{1} e_{1}-E_{3} e_{3} .
$$

(The constant $E_{0}$ could be calculated similarly to $E_{3}$, but we do not need its explicit value.)

Substituting (121)-(125) into (119), we obtain

$$
\mathcal{I}_{1} f=c \varepsilon^{3} \wp^{\prime}(x)\left\{45 \varepsilon\left[\zeta(x)-\zeta\left(\omega_{2}\right)\right]+6 G_{1}\left[\zeta\left(x-\omega_{1}\right)-\zeta\left(\omega_{1}\right)\right]\right.
$$

$\left.+6 G_{3}\left[\zeta\left(x-\omega_{3}\right)-\zeta\left(\omega_{3}\right)\right]+\left[-15 \varepsilon+\left(c-1-4 \varepsilon^{2}\right)(2 \varepsilon)^{-1}-6 G_{1} e_{1}-6 G_{3} e_{3}\right]\left(x-\omega_{2}\right)\right\}$, where $x=\varepsilon z$ and

$$
G_{j}=-\frac{B_{j}\left(c-1-4 \varepsilon^{2}\right)}{48 \varepsilon}-\frac{5 \varepsilon}{24} E_{j}-\frac{\varepsilon C(\varepsilon)}{12} D_{j}, \quad j=1,3 .
$$

3.7. The limit of iterations as $\varepsilon \rightarrow 0$. In this subsection we assume that conditions of Theorem 2.1 hold. In the $\varepsilon=0$ case, direct calculation, based on (50) and (49), shows that

$$
\Delta w_{1}(z, 0)=-\frac{90}{z^{4}}+\frac{12 \cdot 6 !}{7}\left(-z^{-3} \int_{\infty}^{z} t^{2} \mathcal{I}_{2}\left[t^{-6}\right] d t+z^{4} \int_{\infty}^{z} t^{-5} \mathcal{I}_{2}\left[t^{-6}\right] d t\right) .
$$

Copyright $@$ by SIAM. Unauthorized reproduction of this article is prohibited. 
It is easy to check that $\Delta w_{n}, n=2,3, \ldots$, is a function, analytic in $T_{z_{1}}$ and possessing an asymptotic expansion in powers of $z^{-2}$ there, such that

$$
\Delta w_{n}(z) \sim O\left(z^{-2(n+1)}\right), \quad z \rightarrow \infty,|\arg z|<\frac{\pi}{2} .
$$

Then, according to Corollary 1.3 , the solution (34) to (23) in the case $\varepsilon=0$ coincides with $v_{+}(z)$, i.e., $v(z, 0)=v_{+}(z)$.

Let $w(z, \varepsilon)$ denote the solution (54) from Theorem 2.1. In order to prove the continuity of $w(z, \varepsilon)$ in $\varepsilon$ at $\varepsilon=0$, we need the following statement.

Proposition 3.12. Let $g(z, \varepsilon)$ be analytic in $z$ in $T_{z_{1}}$ and continuous in $\varepsilon$ for all sufficiently small $\varepsilon \geq 0$. Moreover, let $g(z, 0) \sim O\left(z^{-b}\right)$ and $\Delta_{\varepsilon} g(z, \varepsilon) \sim O\left(z^{-a}\right)$ in $T_{z_{1}}$, the latter uniformly in $\varepsilon$, where $a, b \geq 0$ and $\Delta_{\varepsilon} g(z, \varepsilon)=g(z, \varepsilon)-g(z, 0)$. Then

$$
\Delta_{\varepsilon} \mathcal{I}_{2} g(z, \varepsilon)=O\left(z^{-a}\right)+O\left(\varepsilon^{b}\right)
$$

and, if $b>5$,

$$
\Delta_{\varepsilon} \mathcal{I}_{1} g(z, \varepsilon)=O\left(z^{2-a}\right)+O\left(\varepsilon^{b-5} z^{-3}\right)+O\left(z^{-b+4} \varepsilon^{2}\right),
$$

where both (130) and (131) are uniform in $T_{z_{1}}$ and in small $\varepsilon \geq 0$.

Proof. Since $g(z, \varepsilon)$ is analytic in $T_{z_{1}}$,

$$
\begin{aligned}
\Delta_{\varepsilon} \mathcal{I}_{2} g(z, \varepsilon)= & \frac{1}{2 i}\left[\int_{\tilde{\omega}_{1}-\tilde{\omega}_{3}}^{z} e^{i(z-\xi)} \Delta_{\varepsilon} g(\xi, \varepsilon) d \xi-\int_{\tilde{\omega}_{1}+2 \tilde{\omega}_{3}}^{z} e^{-i(z-\xi)} \Delta_{\varepsilon} g(\xi, \varepsilon) d \xi\right] \\
& +\frac{1}{2 i}\left[\int_{\infty}^{\tilde{\omega}_{1}-\tilde{\omega}_{3}} e^{i(z-\xi)} g(\xi, 0) d \xi-\int_{\infty}^{\tilde{\omega}_{1}+2 \tilde{\omega}_{3}} e^{-i(z-\xi)} g(\xi, 0) d \xi\right]
\end{aligned}
$$

According to Lemma 3.5, estimate (130) follows directly (132).

To prove (131), we first consider $\Delta_{\varepsilon} q^{2}(z, \varepsilon)$. Representing $\wp(x)=\left[\wp(x)-\frac{1}{x^{2}}\right]+$ $\frac{1}{x^{2}}=\Psi(x)+\frac{1}{x^{2}}$, we note that $\Psi(x)$ is analytic in the trapezoidal region $\tilde{T}$ (vertices at $\omega_{1}-\omega_{3}, \omega_{1}+2 \omega_{3}, \frac{3}{2} \omega_{3},-\frac{1}{2} \omega_{3}$ ). (The trapezoid $\tilde{T}$ is the image of the trapezoid $T$ under the scaling $x=\varepsilon z$.) Since $q^{2}(z, 0)=\frac{1}{z^{2}}$ for any $z \in T$, we have

$$
\Delta_{\varepsilon} q^{2}(z, \varepsilon)=\varepsilon^{2}\left[\Psi(\varepsilon z)-\frac{1}{12}\right]=O\left(\varepsilon^{2}\right)
$$

uniformly in $T$. Similarly, using (45), (46), and (71), we obtain

$$
v_{1}(z, \varepsilon)-v_{1}(z, 0)=O\left(\varepsilon^{3}\right)
$$

and

$$
v_{2}(z, \varepsilon)-v_{2}(z, 0)=O\left(\varepsilon^{2} z^{6}\right)
$$

uniformly in $T$.

Copyright $@$ by SIAM. Unauthorized reproduction of this article is prohibited. 
Now,

(136)

$$
\begin{aligned}
\Delta_{\varepsilon} \mathcal{I}_{1} g(z, \varepsilon)= & -\left[v_{1}(z, \varepsilon) \int_{\tilde{\omega}_{2}}^{z} v_{2}(\xi, \varepsilon) g(\xi, \varepsilon) d \xi-v_{1}(z, 0) \int_{\tilde{\omega}_{2}}^{z} v_{2}(\xi, 0) g(\xi, 0) d \xi\right] \\
& +\left[v_{2}(z, \varepsilon) \int_{\tilde{\omega}_{2}}^{z} v_{1}(\xi, \varepsilon) g(\xi, \varepsilon) d \xi-v_{2}(z, 0) \int_{\tilde{\omega}_{2}}^{z} v_{1}(\xi, 0) g(\xi, 0) d \xi\right] \\
& +\left[-v_{1}(z, 0) \int_{\infty}^{\tilde{\omega}_{2}} v_{2}(\xi, 0) g(\xi, 0) d \xi+v_{2}(z, 0) \int_{\infty}^{\tilde{\omega}_{2}} v_{1}(\xi, 0) g(\xi, 0) d \xi\right] .
\end{aligned}
$$

The third term in the right-hand side of (136) is of the order $O\left(\varepsilon^{b-2}\right)$ as $\varepsilon \rightarrow 0$, while the first term can be represented as

$$
\begin{aligned}
& -v_{1}(z, \varepsilon) \int_{\tilde{\omega}_{2}}^{z}\left[v_{2}(\xi, \varepsilon) \Delta_{\varepsilon} g(\xi, \varepsilon)+g(\xi, 0) \Delta_{\varepsilon} v_{2}(\xi, \varepsilon)\right] d \xi \\
& -\Delta_{\varepsilon} v_{1}(z, \varepsilon) \int_{\tilde{\omega}_{2}}^{z} v_{2}(\xi, 0) g(\xi, 0) d \xi
\end{aligned}
$$

Using Lemma 2.4 together with (134), (135), we obtain estimates

$$
O\left(z^{2-a}\right)+O\left(\varepsilon^{2} z^{4-b}\right)+O\left(\varepsilon^{3} z^{5-b}\right)
$$

for the three terms of (137) that are uniform in $T$ and in small $\varepsilon \geq 0$. The second term in the right-hand side of (136) yields the same result. Now (131) follows from (138) and (94).

THEOREM 3.13. (1) If in condition (53) of Theorem 2.1 we require $\alpha>1$, then

$$
\lim _{\varepsilon \rightarrow 0} v(z, \varepsilon)=v_{+}(z)
$$

uniformly on compact subsets of $T_{z_{1}}$.

(2) If condition (53) of Theorem 2.1 is replaced by

$$
c(\varepsilon)=1+\varepsilon^{\alpha} \tilde{c}(\varepsilon) \quad \text { and } \quad b(\varepsilon)=\varepsilon^{\alpha} \tilde{b}(\varepsilon),
$$

where $\alpha=1$ and functions $\tilde{c}(\varepsilon), \tilde{b}(\varepsilon)$ are continuous for small $\varepsilon \geq 0$, then

$$
\lim _{\varepsilon \rightarrow 0} v(z, \varepsilon)=v_{+}\left(z-z_{*}\right)
$$

uniformly on compact subsets of $T_{z_{1}}$, where the translation $z_{*}$ depends on $\tilde{b}(0), \tilde{c}(0)$. Proof. (1) As a consequence of (133), we obtain that (139) is equivalent to

$$
\lim _{\varepsilon \rightarrow 0} w(z, \varepsilon)=w(z, 0)
$$

as $\varepsilon \rightarrow 0$ uniformly on compact subsets of $T_{z_{1}}$. Since $w(z, \varepsilon)=\sum_{n=1}^{\infty} \Delta w_{n}(z, \varepsilon)$, where the series converges uniformly for all sufficiently small $\varepsilon \geq 0$ and all $z \in T_{z_{1}}$, it is sufficient to prove the continuity of each $\Delta w_{n}(z, \varepsilon)$ in $\varepsilon \geq 0$ in order to prove the continuity of $w(z, \varepsilon)$.

Copyright (c) by SIAM. Unauthorized reproduction of this article is prohibited. 
According to (36) and (94),

$$
f(z, 0)=-6 ! z^{-6} \quad \text { and } \quad \Delta_{\varepsilon} f(z, \varepsilon)=O\left(\varepsilon^{\alpha} z^{-4}\right) .
$$

Then, according to Proposition 3.12,

$$
\Delta_{\varepsilon} \mathcal{I}_{1} f(z, \varepsilon)=O\left(\varepsilon^{\tilde{\alpha}} z^{-3}\right),
$$

where $\tilde{\alpha}=\min \{\alpha-1,1\}$. Using

$$
\Delta_{\varepsilon}[h(z, \varepsilon) g(z, \varepsilon)]=h(z, \varepsilon) \Delta_{\varepsilon} g(z, \varepsilon)+\Delta_{\varepsilon} h(z, \varepsilon) g(z, 0)
$$

together with (36) and (130), we prove that $\Delta w_{1}(z, \varepsilon)=w_{1}(z, \varepsilon)$ also satisfies (144).

Now $\lim _{\varepsilon \rightarrow 0} \Delta w_{n}(z, \varepsilon)=\Delta w_{n}(z, 0), n=2,3, \ldots$, can be proved by induction. Assume that $\Delta_{\varepsilon}\left(\Delta w_{k}(z, \varepsilon)\right)$ satisfies (144) for every $k \in \mathbb{N}, k \leq n$. We want to prove the statement for $k=n+1$. According to (94), (129), and (130), $\Delta_{\varepsilon} g(z, \varepsilon)=$ $O\left(\varepsilon^{\tilde{\alpha}} z^{-6}\right)$, where $g(z, \varepsilon)$ denotes the argument of $\mathcal{I}_{1}$ in $(100)$. It is easy to see that $g(z, 0)=O\left(z^{-2(n+3)}\right)$. Then the fact that $\Delta_{\varepsilon}\left[\Delta w_{n+1}(z, \varepsilon)\right]$ satisfies (144) follows from (131). The proof of part (1) is completed.

(2) According to (126), for a given $z \in T_{z_{1}}$

$$
\lim _{\varepsilon \rightarrow 0} \mathcal{I}_{1} f=-\frac{90}{z^{4}}+\frac{\tilde{c}(0)\left\{\sum_{j=1,3} B_{j}\left[2 \zeta\left(\omega_{j}\right)-\omega_{2} e_{j}\right]-4 \omega_{2}\right\}}{4 z^{3}},
$$

where $B_{j}$ are defined by (122). Similarly,

$$
\lim _{\varepsilon \rightarrow 0} \varepsilon^{7} \tilde{b}(\varepsilon) v_{22}=-\tilde{b}(0) \frac{\frac{3 g_{3}}{2} \omega_{2}-g_{2} \zeta\left(\omega_{2}\right)}{3 \Delta z^{3}} .
$$

Thus,

$$
\lim _{\varepsilon \rightarrow 0} w_{1}(z, \varepsilon)=-\frac{90}{z^{4}}+\frac{12 z_{*}}{z^{3}},
$$

where the constant $z_{*}$, which depend on $\tilde{b}(0), \tilde{c}(0)$, can be derived from (146), (147).

Now we can repeat the argument of part (1) to show that $\Delta w_{n}(z, \varepsilon)$ is continuous in $\varepsilon$. It is pretty straightforward to show that $\Delta w_{n}(z, 0)=O\left(z^{-n-2}\right)$ as $z \rightarrow \infty$ and that $\Delta w_{n}(z, 0)$ has asymptotic expansion in $z^{-1}$. Due to uniform convergence of series (54) in Theorem 2.1, we proved that $\lim _{\varepsilon \rightarrow 0} v(z, \varepsilon)$ is a solution of the truncated equation (24) that has asymptotic expansion in $z^{-1}$ as $z \rightarrow \infty$ with the leading terms $\frac{6}{z^{2}}+\frac{12 z_{*}}{z^{3}}+\cdots$. Then (141) follows from Corollary 1.3.

4. Nonexistence of symmetric periodic solutions to (3). Let $S$ be an interval of $\mathbb{R}$ that contains the point $\omega_{1}=\omega_{1}(0)$, and let $y(x, \varepsilon)$ be a $C^{\alpha}$-deformation of $y(x, 0)=6 \wp_{\frac{1}{12}}, g_{3}\left(x-\omega_{3}\right)-\frac{1}{2}$, where $\left|g_{3}\right|<6^{-3}$, and where $\omega_{1}, \omega_{3}$, and $g_{3}$ are related through (9). As mentioned earlier, it is more convenient for us to consider $y(x, \varepsilon)$ as a $C^{\alpha}$-deformation of $y(x, 0)=6 \wp_{\frac{1}{12}}, g_{3}(x)-\frac{1}{2}$ on the interval $S+\omega_{3}$. Assume that $y(x, \varepsilon)$ contains a sequence $\left\{y\left(x, \varepsilon_{m}\right)\right\}_{1}^{\infty}$ of solutions that are symmetrical at $x=\omega_{3}\left(\varepsilon_{m}\right)$ (after a proper translation $\beta(\varepsilon)$ ) and periodic (along the horizontal line $\left.x=\omega_{3}\left(\varepsilon_{m}\right)+\mathbb{R}\right)$ with the period $2 \omega_{1}\left(\varepsilon_{m}\right)$, where $\lim _{m \rightarrow \infty} \varepsilon_{m}=0$ and $\omega_{1}\left(\varepsilon_{m}\right)$ satisfy (14). Here $\omega_{1}(\varepsilon), \omega_{3}(\varepsilon)$, and $g_{3}(\varepsilon)$ are related through $(9)$.

Let us show that $\beta\left(\varepsilon_{m}\right)=O\left(\varepsilon_{m}^{\alpha}\right)$. Indeed, since $y(x, \varepsilon)$ is a $C^{\alpha}$-deformation of $y(x, 0)$, we know that $y^{\prime}\left(\omega_{2}(\varepsilon)+\beta(\varepsilon), \varepsilon\right)-y^{\prime}\left(\omega_{2}(\varepsilon)+\beta(\varepsilon), 0\right)=O\left(\varepsilon^{\alpha}\right)$ and $\lim _{\varepsilon \rightarrow 0} \beta(\varepsilon)=$ 
0 . But solutions $y\left(x+\beta\left(\varepsilon_{m}\right), \varepsilon_{m}\right)$ are symmetrical at $\omega_{3}\left(\varepsilon_{m}\right)$ and have period $2 \omega_{1}\left(\varepsilon_{m}\right)$, $m \in \mathbb{N}$, so $y^{\prime}\left(\omega_{2}\left(\varepsilon_{m}\right)+\beta\left(\varepsilon_{m}\right), \varepsilon_{m}\right)=0$. Thus,

$$
y^{\prime}\left(\omega_{2}\left(\varepsilon_{m}\right)+\beta\left(\varepsilon_{m}\right), 0\right)=O\left(\varepsilon_{m}^{\alpha}\right) .
$$

Since $y^{\prime}\left(\omega_{2}, 0\right)=0$, then $y^{\prime}\left(\omega_{2}\left(\varepsilon_{m}\right)+\beta\left(\varepsilon_{m}\right), 0\right)=\beta\left(\varepsilon_{m}\right) y^{\prime \prime}\left(\omega_{2}\left(\varepsilon_{m}\right)+\theta\left(\varepsilon_{m}\right) \beta\left(\varepsilon_{m}\right), 0\right)$ by the mean value theorem, where $\theta\left(\varepsilon_{m}\right) \in(0,1)$. Combining this with (149) and the fact that $\wp^{\prime \prime}$ is separated from zero in a vicinity of $\omega_{2}$ (see (64)), we obtain $\beta\left(\varepsilon_{m}\right)=O\left(\varepsilon_{m}^{\alpha}\right)$. Since derivatives of $y(x, 0)$ are bounded on the line $\omega_{3}+\mathbb{R}$, it is easy to show that $y\left(x+\beta\left(\varepsilon_{m}\right), \varepsilon_{m}\right)$ are also $C^{\alpha}$-deformations of $y(x, 0)$. Thus, without any loss of generality, we will consider only such deformations $y(x, \varepsilon)$ of $y(x, 0)$ that are symmetrical at $\omega_{2}(\varepsilon)$. That means that solutions $v(z, \varepsilon)$ to the inner equation (4), that correspond to such $y(x, \varepsilon)$, are symmetrical at $\tilde{\omega}_{2}(\varepsilon)$.

Let us denote by $\mathcal{F}_{\alpha}, \alpha \geq 1$, the two-parameter family of solutions constructed in Theorem 2.1 that satisfy an additional assumption: condition (53) in Theorem 2.1 is replaced by (140), where $\alpha \geq 1$. The proof of Theorem 1.1 is divided into the following two steps: (1) if $v(z, \varepsilon)$ is the inner solution, symmetrical at $z=\tilde{\omega}_{2}(\varepsilon)$, that corresponds to a $C^{\alpha}$-deformation $y(x, \varepsilon)$ and if $\omega_{1}(\varepsilon)$ satisfies $(14)$, then $v(z, \varepsilon) \in \mathcal{F}_{\alpha}$; (2) if $\omega_{1}(\varepsilon)$ satisfies $(14)$, then there is no symmetrical and $2 \omega_{1}(\varepsilon)$ periodic solution $v(z, \varepsilon) \in \mathcal{F}_{\alpha}$. Here and below we use $\tilde{\omega}_{j}$ to denote $\tilde{\omega}_{j}(\varepsilon), j=1,2,3$, wherever such notation is clear. Additionally, we prove that the BVP

$$
\begin{aligned}
2 v^{\prime \prime \prime} v^{\prime}-v^{\prime \prime 2}+\left(1-\varepsilon^{2}\right) v^{\prime 2}-\varepsilon^{2} v^{2}-\frac{2}{3} v^{3} & =\varepsilon^{6} C(\varepsilon), \\
v^{\prime}\left(\tilde{\omega}_{3}, \varepsilon\right) & =v^{\prime}\left(\tilde{\omega}_{2}, \varepsilon\right)=v^{\prime \prime \prime}\left(\tilde{\omega}_{2}, \varepsilon\right)=0
\end{aligned}
$$

has a unique solution in $\mathcal{F}_{\alpha}$.

If $y(x, \varepsilon)$ is a $C^{\alpha}$-deformation of solution (16), then the constant of motion $C(\varepsilon)$ of $y(x, \varepsilon)$, given by (11), satisfies (13). Then the corresponding inner solution $v(z, \varepsilon)$ satisfies (150) and the two latter boundary conditions of (150). It is easy to see that $v(z, \varepsilon)$ is periodic with the period $2 \omega_{1}(\varepsilon)$ and symmetrical if and only if, additionally, $v^{\prime}\left(\tilde{\omega}_{3}, \varepsilon\right)=v^{\prime \prime \prime}\left(\tilde{\omega}_{3}, \varepsilon\right)=0$. In other words, a solution $v(z, \varepsilon)$ to the BVP (150) is symmetric and periodic with the period $2 \omega_{1}(\varepsilon)$ if and only if $v^{\prime \prime \prime}\left(\tilde{\omega}_{3}, \varepsilon\right)=0$.

4.1. Solution of BVP (150). Here we prove existence and uniqueness of solution to BVP (150) within the family $\mathcal{F}_{\alpha}$ using the implicit function theorem.

If $v(z, \varepsilon)$ is a solution to the BVP (150), then the integral of motion (150) evaluated at the points $z=\tilde{\omega}_{2}, z=\tilde{\omega}_{3}$ becomes

$$
\varepsilon^{2} v^{2}\left(\tilde{\omega}_{j}\right)+\frac{2}{3} v^{3}\left(\tilde{\omega}_{j}\right)+v^{\prime \prime 2}\left(\tilde{\omega}_{j}\right)+\varepsilon^{6} C(\varepsilon)=0
$$

where $j=2,3$. We want to show that for sufficiently small $\varepsilon$ there exists a unique $v \in \mathcal{F}_{\alpha}$ satisfying (151). To this end, we can restrict our attention on the triangle $P \subset T_{z_{1}}$, which was introduced in section 3.4.

According to (34), (49),

$$
v=6 q^{2}+\tilde{v}=6 q^{2}+6 \varepsilon^{\alpha} \tilde{c} q^{2}+\mathcal{I}_{2}\left[\varepsilon^{6+\alpha} \tilde{b} v_{22}+\mathcal{I}_{1} f(q)+\tilde{w}\right]
$$

where

$$
\tilde{w}=-12 c \mathcal{I}_{1} q^{2} \mathcal{I}_{2} f(q)+\sum_{n=2}^{\infty} \Delta w_{n}
$$

Copyright $@$ by SIAM. Unauthorized reproduction of this article is prohibited. 
It is easy to see that $v(z, \varepsilon)=O\left(\varepsilon^{2}\right)$ uniformly in $P$ as $\varepsilon \rightarrow 0$. According to (106), (140), and Remark 3.8, we have $w(z, \varepsilon)=O\left(\varepsilon^{3+\tilde{\alpha}}\right)$ uniformly in $P$, where $\tilde{\alpha}=\min \{1, \alpha-1\}$ as $\varepsilon \rightarrow 0$. Then, according to (152) and Lemma 2.4,

$$
\tilde{v} \sim O\left(\varepsilon^{3+\tilde{\alpha}}\right) \text { and } \tilde{w} \sim O\left(\varepsilon^{4+2 \tilde{\alpha}}\right)
$$

uniformly in $P$ as $\varepsilon \rightarrow 0$. Now combining (154) and (39), we see that

$$
w^{\prime \prime}=\varepsilon^{2} w+\left[\mathcal{I}_{2} w\right]^{2}+12 c q^{2} \mathcal{I}_{2} w+f(q) \sim O\left(\varepsilon^{5+\tilde{\alpha}}\right) \quad \text { as } \quad \varepsilon \rightarrow 0,
$$

uniformly on $P$. Then

$$
w^{\prime}(z, \varepsilon)=\int_{\tilde{\omega}_{2}}^{z} w^{\prime \prime}(t, \varepsilon) d t=O\left(\varepsilon^{4+\tilde{\alpha}}\right)
$$

uniformly in $z \in P$. To estimate $v^{\prime \prime}$ in (151) we need the following statement.

Proposition 4.1. Let $g(z, \varepsilon)$ be a differentiable in $z \in T_{z_{1}}$ function. If there exists a constant $M>0$ such that for all small $\varepsilon>0$ both $|g(z, \varepsilon)|$ and $\left|g^{\prime}(z, \varepsilon)\right|$ are bounded by $M$ in $T_{z_{1}}$, then there exists some $\delta_{0}>0$ such that

$$
\mathcal{I}_{2} g(z, \varepsilon)=g(z, \varepsilon)-\mathcal{I}_{2} g^{\prime \prime}(z, \varepsilon)+O\left(e^{-\frac{\delta_{0}}{\varepsilon} \rho(z)}\right)
$$

as $\varepsilon \rightarrow 0$ uniformly in $P$, where $\rho(z)=\min \left\{\left|z-\left(\tilde{\omega}_{1}+3 \tilde{\omega}_{3}\right)\right|,\left|z-\left(\tilde{\omega}_{1}-\tilde{\omega}_{3}\right)\right|\right\}$.

Proof. Integrating by parts $\mathcal{I}_{2} g(z, \varepsilon)$, expressed by (76) and (83), twice, we obtain

$$
\begin{aligned}
\mathcal{I}_{2} g(z, \varepsilon) & =g(z, \varepsilon)-\mathcal{I}_{2} g^{\prime \prime}(z, \varepsilon)-\left.\frac{1}{2} e^{i(z-\xi)}\left[g(\xi, \varepsilon)-i g^{\prime}(\xi, \varepsilon)\right]\right|_{\xi=\tilde{\omega}_{1}-\tilde{\omega}_{3}} \\
& -\left.\frac{1}{2} e^{-i(z-\xi)}\left[g(\xi, \varepsilon)+i g^{\prime}(\xi, \varepsilon)\right]\right|_{\xi=\tilde{\omega}_{1}+3 \tilde{\omega}_{3}} .
\end{aligned}
$$

The last two terms of (158) are exponentially small in $\varepsilon^{-1}$ according to the construction of $T_{z_{1}}$.

Under the assumptions of Proposition 4.1,

$$
D^{2} \mathcal{I}_{2} g=g-\mathcal{I}_{2} g=\mathcal{I}_{2} D^{2} g+O\left(e^{-\frac{\delta_{0}}{\varepsilon} \rho(z)}\right) .
$$

According to (156), Proposition 4.1 is applicable for $g(z, \varepsilon)=w(z, \varepsilon)$. Thus,

$$
v^{\prime \prime}=6 c(\varepsilon)\left(q^{2}\right)^{\prime \prime}+\mathcal{I}_{2} w^{\prime \prime}=O\left(\varepsilon^{4}\right) \quad \text { as } \quad \varepsilon \rightarrow 0,
$$

uniformly on $\left[\tilde{\omega}_{3}, \tilde{\omega}_{2}\right]$.

Note that $y=\frac{6 q^{2}}{\varepsilon^{2}}$ is the solution of the unperturbed equation (2) with the constant of motion $J(\varepsilon)$; see (37). Thus,

$$
\left(\frac{6 q^{2}}{\varepsilon^{2}}\right)^{2}+\frac{2}{3}\left(\frac{6 q^{2}}{\varepsilon^{2}}\right)^{3}+J(\varepsilon)=0
$$

at $x=\varepsilon z=\omega_{j}, j=1,2,3$. Notice that (9) and (14) imply that

$$
J(\varepsilon)=C+\varepsilon^{\alpha} \tilde{J}(\varepsilon)
$$

Copyright (c) by SIAM. Unauthorized reproduction of this article is prohibited. 
where $\tilde{J}(\varepsilon)$ is a continuous function. Now substituting $v=6 q^{2}+\tilde{v}$ into (151), dividing both sides by $\varepsilon^{7+\tilde{\alpha}}$, and taking into account (161), we obtain

$$
\begin{aligned}
2\left(\frac{6 q^{2}}{\varepsilon^{2}}\right) \breve{v} & +\varepsilon^{1+\tilde{\alpha}} \breve{v}^{2}+2\left(\frac{6 q^{2}}{\varepsilon^{2}}\right)^{2} \breve{v}+2 \varepsilon^{1+\tilde{\alpha}}\left(\frac{6 q^{2}}{\varepsilon^{2}}\right) \breve{v}^{2} \\
& +\frac{2}{3} \varepsilon^{2+2 \tilde{\alpha}} \breve{v}^{3}+\frac{v^{\prime \prime 2}}{\varepsilon^{7+\tilde{\alpha}}}+\tilde{C}(\varepsilon)-\tilde{J}(\varepsilon)=0
\end{aligned}
$$

at $z=\tilde{\omega}_{2}, \tilde{\omega}_{3}$, where

$$
\breve{v}(z, \varepsilon)=\frac{\tilde{v}(z, \varepsilon)}{\varepsilon^{3+\tilde{\alpha}}}=\tilde{c}(\varepsilon)\left(\frac{6 q^{2}}{\varepsilon^{2}}\right)+\varepsilon^{4} \tilde{b}(\varepsilon) \mathcal{I}_{2} v_{22}+\mathcal{I}_{2} \frac{\mathcal{I}_{1} f(q)+\tilde{w}}{\varepsilon^{3+\tilde{\alpha}}} .
$$

Equation (163) evaluated at the points $z=\tilde{\omega}_{2}, \tilde{\omega}_{3}$ forms a system that we denote by

$$
F(\varepsilon, \tilde{b}, \tilde{c})=0 .
$$

LEMMA 4.2. There exist some $\varepsilon_{1} \in\left(0, \varepsilon_{0}\right]$ and functions $\tilde{b}(\varepsilon), \tilde{c}(\varepsilon)$, continuous on $\left[0, \varepsilon_{1}\right]$, such that the system (165) holds identically on $\left[0, \varepsilon_{1}\right]$. Moreover, the solution $\tilde{b}(\varepsilon), \tilde{c}(\varepsilon)$ to $(165)$ is unique.

Proof. The proof is based on the implicit function theorem (see, for example, [MB, p. 122]). According to this theorem, we have to show that (a) $F(0, \tilde{b}(0), \tilde{c}(0))=0$ for some $\tilde{b}(0), \tilde{c}(0)$; (b) matrix

$$
\left.\operatorname{Col}\left(\frac{\partial F}{\partial \tilde{b}}, \frac{\partial F}{\partial \tilde{c}}\right)\right|_{\varepsilon=0}
$$

is not singular; and (c) $F$ and $\frac{\partial F}{\partial \tilde{c}}, \frac{\partial F}{\partial \grave{b}}$ are continuous in all variables in a vicinity of $(0, \tilde{b}(0), \tilde{c}(0))$. Then

According to (156), we can apply Proposition 4.1 to $\breve{v}(z, \varepsilon)-\tilde{c}(\varepsilon) \frac{6 q^{2}}{\varepsilon^{2}}=\frac{\mathcal{I}_{2} w}{\varepsilon^{3+\alpha}}$.

(167) $\lim _{\varepsilon \rightarrow 0} \breve{v}(z, \varepsilon)=\tilde{c}(0)\left(6 \wp(\varepsilon z)-\frac{1}{2}\right)+\tilde{b}(0) \lim _{\varepsilon \rightarrow 0} \varepsilon^{4} v_{22}(z, \varepsilon)+\lim _{\varepsilon \rightarrow 0} \frac{\mathcal{I}_{1} f(q)}{\varepsilon^{3+\tilde{\alpha}}}+\lim _{\varepsilon \rightarrow 0} \frac{\tilde{w}}{\varepsilon^{3+\tilde{\alpha}}}$

uniformly on $\left[\tilde{\omega}_{3}, \tilde{\omega}_{2}\right]$. Due to Remark 3.8 , the last limit in (167) is zero.

Using (63), (69), (47), and the fact that $v_{1}\left(\tilde{\omega}_{j}\right)=0$, we have

$$
\lim _{\varepsilon \rightarrow 0} \varepsilon^{4} v_{22}\left(\tilde{\omega}_{j}, \varepsilon\right)=-\frac{1}{12} \frac{1}{\left(e_{j}-e_{i}\right)\left(e_{j}-e_{k}\right)} .
$$

Note that $\mathcal{I}_{1} f(q)\left(\tilde{\omega}_{2}\right)=0$. To calculate $\lim _{\varepsilon \rightarrow 0} \varepsilon^{-3-\tilde{\alpha}} \mathcal{I}_{1} f(q)\left(\tilde{\omega}_{3}\right)$, we notice that

$$
\begin{aligned}
\wp^{\prime}\left(x-\omega_{j}\right) & =\left(6 e_{j}^{2}-\frac{g_{2}}{2}\right)\left(x-\omega_{j}\right)+O\left(\left(x-\omega_{j}\right)^{2}\right) \\
& =2\left(e_{i}-e_{j}\right)\left(e_{k}-e_{j}\right)\left(x-\omega_{j}\right)+O\left(\left(x-\omega_{j}\right)^{2}\right)
\end{aligned}
$$

as $x \rightarrow \omega_{j}, j=1,2,3$. It follows then from (126) that

$$
=\frac{c(\varepsilon)}{e_{1}-e_{3}}\left\{-\frac{c(\varepsilon)-1-4 \varepsilon^{2}}{4 \varepsilon}\left(e_{1}+\frac{1}{6}\right)-\frac{5 \varepsilon}{2}\left(\frac{9}{4} e_{3}+\frac{3}{2} e_{2}+36 e_{2}^{3}-\frac{1}{3}\right)+\varepsilon C(\varepsilon)\right\} .
$$

Copyright (c) by SIAM. Unauthorized reproduction of this article is prohibited. 
Using (126), we obtain

$$
\begin{aligned}
\lim _{\varepsilon \rightarrow 0} \varepsilon^{-3-\tilde{\alpha}} \mathcal{I}_{1} f(q)\left(\tilde{\omega}_{3}=\right. & -\frac{\tilde{c}(\varepsilon)\left(e_{1}+\frac{1}{6}\right)}{4\left(e_{1}-e_{3}\right)} \\
& -\delta_{\tilde{\alpha}, 1} \frac{e_{1}+\frac{1}{6}+\frac{5}{2}\left(\frac{9}{4} e_{3}+\frac{3}{2} e_{2}+36 e_{2}^{3}-\frac{1}{3}\right)+36 g_{3}+\frac{1}{6}}{e_{1}-e_{3}}
\end{aligned}
$$

at $z=\tilde{\omega}_{3}$, where $\delta_{\tilde{\alpha}, 1}=1$ if $\tilde{\alpha}=1$ and $\delta_{\tilde{\alpha}, 1}=0$ otherwise. Combining (167)-(171), we obtain

$$
\begin{aligned}
& \lim _{\varepsilon \rightarrow 0} \breve{v}\left(\tilde{\omega}_{2}\right)=\left(6 e_{2}-\frac{1}{2}\right) \tilde{c}(0)+\frac{\tilde{b}(0)}{12\left(e_{1}-e_{2}\right)\left(e_{2}-e_{3}\right)} \\
& \lim _{\varepsilon \rightarrow 0} \breve{v}\left(\tilde{\omega}_{3}\right)=\left[\left(6 e_{3}-\frac{1}{2}\right)-\frac{e_{1}+\frac{1}{6}}{4\left(e_{1}-e_{3}\right)}\right] \tilde{c}(0)-\frac{\tilde{b}(0)}{12\left(e_{1}-e_{3}\right)\left(e_{2}-e_{3}\right)}-\delta_{\tilde{\alpha}, 1} \tilde{D}
\end{aligned}
$$

where $\tilde{D}$ denotes the last fraction in (171).

According to (154) and (160), in the limit $\varepsilon \rightarrow 0$ equations (163) become

$$
2\left[36 \wp^{2}\left(\omega_{j}\right)-\frac{1}{4}\right] \lim _{\varepsilon \rightarrow 0} \breve{v}\left(\tilde{\omega}_{j}\right)+\tilde{C}(0)-\tilde{J}(0)=0,
$$

where $j=2,3$. Clearly, (172)-(173) form a linear system of equations for the unknowns $\tilde{b}(0), \tilde{c}(0)$. Note that the first factor in (173) is different from zero since, otherwise, according to (64), we would have either $C=0$ or $C=-\frac{1}{3}$, which contradicts our assumption $C \in\left(-\frac{1}{3}, 0\right)$. After some algebra, we calculate the determinant of the matrix of linear system (172)-(173) as

$$
\begin{aligned}
& \left|\begin{array}{cc}
6 e_{2}-\frac{1}{2} & \frac{1}{12\left(e_{1}-e_{2}\right)\left(e_{2}-e_{3}\right)} \\
6 e_{3}-\frac{1}{2}-\frac{e_{1}+\frac{1}{6}}{4\left(e_{1}-e_{3}\right)} & -\frac{1}{12\left(e_{1}-e_{3}\right)\left(e_{2}-e_{3}\right)}
\end{array}\right|=\frac{1}{3 \sqrt{\Delta}}\left|\begin{array}{cc}
\left(6 e_{2}-\frac{1}{2}\right)\left(e_{1}-e_{2}\right) & 1 \\
\left(6 e_{3}-\frac{1}{2}\right)\left(e_{1}-e_{3}\right)-\frac{e_{1}+\frac{1}{6}}{4} & -1
\end{array}\right| \\
& =\frac{7\left(e_{1}+\frac{1}{6}\right)}{12 \sqrt{\Delta}},
\end{aligned}
$$

where $\Delta$, defined by (67), is different from zero. This determinant is different from zero since $e_{1}>0$. Thus, we have established parts (a) and (b) of the lemma; i.e., we have established that $F(0, \tilde{b}(0), \tilde{c}(0))=0$ at $z=\tilde{\omega}_{2}, \tilde{\omega}_{3}$ for some $\tilde{b}(0), \tilde{c}(0)$ and that matrix (166) is nonsingular. Continuity of $F$ follows from Theorem 3.13 and the fact that iterations $\Delta w_{n}(z, \varepsilon)$ in the solution (54) are polynomials in $\tilde{b}(0), \tilde{c}(0)$ of degree not exceeding $n$. Estimates of Theorem 2.1 can be readily adjusted to prove convergence of the series $\sum_{n=1}^{\infty} \frac{\partial \Delta w_{n}(z, \varepsilon)}{\partial \tilde{c}}$ and $\sum_{n=1}^{\infty} \frac{\partial \Delta w_{n}(z, \varepsilon)}{\partial \tilde{b}}$ at $z=\tilde{\omega}_{2}, z=\tilde{\omega}_{3}$. Part (c) and the whole proof are completed.

THEOREM 4.3. If $C(\varepsilon)$ and $\omega_{1}(\varepsilon)$ satisfy (13) and (14), respectively, where $\alpha \geq 1$, then the BVP (150) has a unique solution in $\mathcal{F}_{\alpha}$.

Proof. According to Lemma 4.2, it is sufficient to show that (151) at $z=\tilde{\omega}_{3}$ and the rescaled integral of motion (150) imply $v^{\prime}\left(\tilde{\omega}_{3}\right)=0$. Indeed, addition of these two equations yields

$$
v^{\prime}\left(\tilde{\omega}_{3}\right)\left[2 v^{\prime \prime \prime}\left(\tilde{\omega}_{3}\right)+\left(1-\varepsilon^{2}\right) v^{\prime}\left(\tilde{\omega}_{3}\right)\right]=0 .
$$

Copyright (c) by SIAM. Unauthorized reproduction of this article is prohibited. 
That means that $v^{\prime}\left(\tilde{\omega}_{3}\right)=0$ or $2 v^{\prime \prime \prime}\left(\tilde{\omega}_{3}\right)+\left(1-\varepsilon^{2}\right) v^{\prime}\left(\tilde{\omega}_{3}\right)=0$. According to (34), and since operators $D$ and $D^{2}+1$ commute, this implies $u^{\prime}\left(\tilde{\omega}_{3}\right)=0$ or $2 w^{\prime}\left(\tilde{\omega}_{3}\right)=$ $\left(1+\varepsilon^{2}\right) u^{\prime}\left(\tilde{\omega}_{3}\right)$. If $u^{\prime}\left(\tilde{\omega}_{3}\right)=0$, then $v^{\prime}\left(\tilde{\omega}_{3}\right)=0$, and the proof is completed. The assumption $u^{\prime}\left(\tilde{\omega}_{3}\right) \neq 0$ leads to a contradiction. Indeed, on one hand, we have

$$
u^{\prime}\left(\tilde{\omega}_{3}\right)=\frac{2}{1+\varepsilon^{2}} w^{\prime}\left(\tilde{\omega}_{3}\right),
$$

whereas, on the other hand, according to Proposition 4.1,

$$
u^{\prime}\left(\tilde{\omega}_{3}\right)=\left.\mathcal{I}_{2} w^{\prime}\right|_{z=\tilde{\omega}_{3}}=w^{\prime}\left(\tilde{\omega}_{3}\right)-\left.\mathcal{I}_{2} w^{\prime \prime \prime}\right|_{z=\tilde{\omega}_{3}}+O\left(e^{-\frac{\delta_{0}}{\varepsilon}}\right)
$$

Proposition 4.1 is applicable to $\mathcal{I}_{2} w^{\prime}$ according to (39) and (156). Moreover, differentiating (39), we obtain $w^{\prime \prime \prime}=O\left(\varepsilon^{6+\tilde{\alpha}}\right)$ uniformly on $\left[\tilde{\omega}_{3}, \tilde{\omega}_{2}\right]$. Thus, (177) contradicts (176).

\subsection{Proof of Theorem 1.1.}

Proof. As mentioned in the beginning of section 4, our proof consists of two steps. Step (1) is to show that the inner solution $v(z, \varepsilon)=\varepsilon^{2} y(\varepsilon z, \varepsilon)$, corresponding to a $C^{\alpha}$-deformation $y(x, \varepsilon)$ of the periodic solution $y(x, 0)$, is an $\mathcal{F}_{\alpha}$ solution. Let us consider first the case $n=1$.

As discussed above, we can assume without any loss of generality that $v\left(z, \varepsilon_{m}\right)$ is symmetrical with respect to $z=\tilde{\omega}_{3}\left(\varepsilon_{m}\right)$ and $z=\tilde{\omega}_{2}\left(\varepsilon_{m}\right)$, where $g_{3}\left(\varepsilon_{m}\right)$ and $\omega_{3}\left(\varepsilon_{m}\right)$ are defined by $\omega_{1}\left(\varepsilon_{m}\right)$ through $(9)$. Then $v^{\prime}\left(\tilde{\omega}_{2}\left(\varepsilon_{m}\right), \varepsilon_{m}\right)=v^{\prime \prime \prime}\left(\tilde{\omega}_{2}\left(\varepsilon_{m}\right), \varepsilon_{m}\right)=0$, so that $v\left(z, \varepsilon_{m}\right)$ is defined by initial conditions $v\left(\tilde{\omega}_{2}\left(\varepsilon_{m}\right), \varepsilon_{m}\right)$ and $v^{\prime \prime}\left(\tilde{\omega}_{2}\left(\varepsilon_{m}\right), \varepsilon_{m}\right)$. In order to show that there is a solution in $\mathcal{F}_{\alpha}$ with the abovementioned initial conditions at $z=\tilde{\omega}_{2}\left(\varepsilon_{m}\right)$, we first establish that

$$
\begin{gathered}
v\left(\tilde{\omega}_{2}\left(e_{n}\right), \varepsilon_{m}\right)-\varepsilon_{m}^{2}\left(6 e_{2}(0)-\frac{1}{2}\right)=\varepsilon_{m}^{2+\alpha} \tilde{v}\left(\varepsilon_{m}\right), \\
v^{\prime \prime}\left(\tilde{\omega}_{2}\left(e_{n}\right), \varepsilon_{m}\right)-\varepsilon_{m}^{4}\left(3 e_{2}^{2}(0)-\frac{1}{48}\right)=\varepsilon_{m}^{4+\alpha} \tilde{v}^{\prime \prime}\left(\varepsilon_{m}\right),
\end{gathered}
$$

where $\tilde{v}(\varepsilon), \tilde{v}^{\prime \prime}(\varepsilon)$ are continuous functions. Indeed, according to our assumptions and taking into account (14),

$$
\begin{aligned}
& y\left(\omega_{2}\left(\varepsilon_{m}\right), \varepsilon_{m}\right)-y\left(\omega_{2}, 0\right) \\
& \quad=\left[y\left(\omega_{2}\left(\varepsilon_{m}\right), \varepsilon_{m}\right)-y\left(\omega_{2}\left(\varepsilon_{m}\right), 0\right)\right]+\left[y\left(\omega_{2}\left(\varepsilon_{m}\right), 0\right)-y\left(\omega_{2}, 0\right)\right] \\
& \quad=\varepsilon_{m}^{\alpha} \tilde{y}\left(\omega_{2}\left(\varepsilon_{m}\right), \varepsilon_{m}\right)+\frac{1}{2} \varepsilon_{m}^{\alpha} \tau^{2}\left(\varepsilon_{m}\right) \wp^{\prime \prime}\left(\omega_{2}(0)+\theta\left(\omega_{1}\left(\varepsilon_{m}\right)-\omega_{1}\right)\right),
\end{aligned}
$$

where $\theta \in(0,1)$. Here we used the mean value theorem and the fact that $\wp^{\prime}\left(\omega_{2}(0)\right)=$ 0 . A similar estimate holds for $y^{\prime \prime}\left(\omega_{2}\left(\varepsilon_{m}\right), \varepsilon_{m}\right)-y^{\prime \prime}\left(\omega_{2}, 0\right)$. Then, (178) follows from (179). 
According to (164), equations (178) in the leading order can be written as

$$
\begin{aligned}
& \tilde{c}(\varepsilon)\left(\frac{6 q^{2}}{\varepsilon^{2}}\right)+\varepsilon^{4} \tilde{b}(\varepsilon) \mathcal{I}_{2} v_{22}+\mathcal{I}_{2} \frac{\mathcal{I}_{1} f(q)+\tilde{w}}{\varepsilon^{3+\tilde{\alpha}}} \\
& \quad=\tilde{v}(\varepsilon)-\frac{1}{2} \tau^{2}(\varepsilon) \wp^{\prime \prime}\left(\omega_{2}(0)+\theta\left(\omega_{1}(\varepsilon)-\omega_{1}(0)\right)\right), \\
& \tilde{c}(\varepsilon) D_{z}^{2}\left(\frac{6 q^{2}}{\varepsilon^{2}}\right)+\varepsilon^{4} \tilde{b}(\varepsilon) D_{z}^{2} \mathcal{I}_{2} v_{22}+D_{z}^{2} \mathcal{I}_{2} \frac{\mathcal{I}_{1} f(q)+\tilde{w}}{\varepsilon^{3+\tilde{\alpha}}} \\
& \quad=\tilde{v}^{\prime \prime}(\varepsilon)-\frac{3}{2}\left(e_{2}(\varepsilon)+e_{2}(0)\right) \tau^{2}(\varepsilon) \wp^{\prime \prime}\left(\omega_{2}(0)+\theta\left(\omega_{1}(\varepsilon)-\omega_{1}(0)\right)\right) .
\end{aligned}
$$

According to (167) and Proposition 4.1, in the limit $\varepsilon \rightarrow 0$ equations (180) become

$$
\begin{gathered}
\tilde{c}(0) \lim _{\varepsilon \rightarrow 0} \frac{6 q^{2}}{\varepsilon^{2}}+\tilde{b}(0) \lim _{\varepsilon \rightarrow 0} \varepsilon^{4} v_{22}\left(\tilde{\omega}_{2}(\varepsilon), \varepsilon\right)=\tilde{v}(0)-\frac{1}{2} \tau^{2} \wp^{\prime \prime}\left(\omega_{2}(0)\right), \\
\tilde{c}(0) \lim _{\varepsilon \rightarrow 0}\left[\frac{6 q^{2}}{\varepsilon^{2}}+\frac{36 q^{4}}{\varepsilon^{4}}\right]+\tilde{b}(0) \lim _{\varepsilon \rightarrow 0}\left\{\varepsilon^{4} v_{22}\left(\tilde{\omega}_{2}(\varepsilon), \varepsilon\right)\left[1+\frac{12 q^{2}}{\varepsilon^{2}}\right]\right\}=\tilde{v}^{\prime \prime}(0) \\
-3 e_{2}(0) \tau^{2}(0) \wp^{\prime \prime}\left(\omega_{2}(0)\right) .
\end{gathered}
$$

Here we used the fact that $\frac{6 q^{2}}{\varepsilon^{2}}$ and $v_{22}$ satisfy differential equations (2) and (44), respectively. Calculation of the determinant of the latter system yields

$$
\begin{gathered}
\lim _{\varepsilon \rightarrow 0}\left|\begin{array}{cc}
\frac{6 q^{2}}{\varepsilon^{2}} & \varepsilon^{4} v_{22} \\
\frac{6 q^{2}}{\varepsilon^{2}}+\frac{36 q^{4}}{\varepsilon^{4}} & {\left[1+\frac{12 q^{2}}{\varepsilon^{2}}\right] \varepsilon^{4} v_{22}}
\end{array}\right|=\lim _{\varepsilon \rightarrow 0}\left|\begin{array}{cc}
\frac{6 q^{2}}{\varepsilon^{2}} & \varepsilon^{4} v_{22} \\
\frac{36 q^{4}}{\varepsilon^{4}} & 12 q^{2} \varepsilon^{2} v_{22}
\end{array}\right| \\
=36 \lim _{\varepsilon \rightarrow 0} q^{4} v_{22}=\frac{3\left(e_{2}(0)-\frac{1}{2}\right)^{2}}{\left(e_{1}(0)-e_{2}(0)\right)\left(e_{2}(0)-e_{3}(0)\right)} \neq 0 .
\end{gathered}
$$

Following the arguments of Lemma 4.2, we can now use the implicit function theorem to show that the system (180) has a unique solution $\tilde{c}(\varepsilon), \tilde{b}(\varepsilon)$, where $\tilde{c}(\varepsilon), \tilde{b}(\varepsilon)$ are continuous functions. Thus, $v(z, \varepsilon) \in \mathcal{F}_{\alpha}$. To prove step (1) for the case for general $n$ we simply have to consider the region $\mathcal{P}$ from Remark 3.10 instead of the triangle $P$ and the point $n \tilde{\omega}_{1}(\varepsilon)+\tilde{\omega}_{3}(\varepsilon)$ instead of $\tilde{\omega}_{2}(\varepsilon)$.

To prove step (2), we assume that there is a family of solutions $v\left(z, \varepsilon_{m}\right) \subset \mathcal{F}_{\alpha}$ to $(4)$, where $\omega_{1}(\varepsilon)$ satisfies $(14)$, that is symmetrical at $\tilde{\omega}_{3}\left(\varepsilon_{m}\right)$. However, according to Theorem 3.13,

$$
\lim _{m \rightarrow \infty} v\left(z, \varepsilon_{m}\right)=v_{+}(z)
$$

uniformly in $z$ on any closed segment of the imaginary axis that belongs to $T_{z_{1}}$. Due to the symmetry with respect to the imaginary axis, we also have

$$
\lim _{m \rightarrow \infty} v\left(z, \varepsilon_{m}\right)=v_{-}(z)
$$

uniformly in $z$ on the same segment of the imaginary axis. But, according to Corollary $1.3, v_{+}(z) \not \equiv v_{-}(z)$. The obtained contradiction proves nonexistence of a family of symmetric periodic solutions $v\left(z, \varepsilon_{m}\right) \subset \mathcal{F}_{\alpha}, \alpha>1$, where $\varepsilon_{m} \rightarrow 0$. The same result, according to Theorem 3.13, part (2), holds for the case $\alpha=1$. 


\section{REFERENCES}

[AM] C. J. Amick And J. B. MCLeOd, A singular perturbation problem in water waves, Stability Appl. Anal. Contin. Media, 1 (1991), pp. 127-148.

[BPBA] R. E. Beardmore, M. A. Peletier, C. J. Budd, And M. Ahmer Wadee, Bifurcations of periodic solutions satisfying the zero-Hamiltonian constraint in reversible differential equations, SIAM J. Math. Anal., 36 (2005), pp. 1461-1488.

[Eck] W. EckHAus, Singular perturbations of homoclinic orbits in $\mathbb{R}^{4}$, SIAM J. Math. Anal., 23 (1992), pp. 1269-1290.

[GR] I. S. Gradsteyn And I. W. Ryzhik, Tables of Integrals, Series and Products, Academic Press, New York, 1965.

[GJ] R. GRImShaW And N. Joshi, Weakly nonlocal solitary waves in a singularly perturbed Korteweg-de Vries equation, SIAM J. Appl. Math., 55 (1995), pp. 124-135.

[Ha] H. Hancock, Theory of Elliptic Functions, Dover, New York, 1958.

[HM] J. M. Hammersley and G. Mazzarino, Computational aspects of some autonomous differential equations, Proc. Roy. Soc. London Ser. A, 424 (1989), pp. 19-37.

[IL1] G. Iooss AND E. Lombard, Normal forms with exponentially small remainder: Application to homoclinic connections for the reversible $0^{2+} i \omega$ resonance, C. R. Math. Acad. Sci. Paris, 339 (2004), pp. 831-838.

[IL2] G. IOOSS AND E. LOMBARDI, Normal forms with exponentially small remainder for analytic vector fields, J. Differential Equations, 212 (2005), pp. 1-61.

[KS] M. D. Kruskal And H. Segur, Asymptotics beyond all orders in a model of crystal growth, Stud. Appl. Math., 85 (1991), pp. 129-181.

[Lo] E. Lombardi, Oscillatory Integrals and Phenomena Beyond All Algebraic Orders. With Applications to Homoclinic Orbits in Reversible Systems, Lecture Notes in Math. 1741, Springer-Verlag, Berlin, 2000.

[MB] E. J. McShane And T. A. Botts, Real Analysis, Van Nostrand Company, New York, 1959.

[PT] L. A. Peletier and W. C. Troy, Spatial Patterns: Higher Order Models in Physics and Mechanics, Birkhäuser Boston, Boston, 2001.

[PRG] Y. Pomeau, A. Ramani, and B. Grammaticos, Structural stability of the Korteweg-de Vries solutions under a singular perturbation, Phys. D, 31 (1988), pp. 127-134.

[STL] H. Segur, S. Tanveer, and H. Levine, eds., Asymptotics beyond All Orders, Nato ASI Ser. B. Phys. 284, Plenum, New York, 1991.

[To2] A. TovBIS, Asymptotics beyond all orders and analytic properties of inverse Laplace transforms of solutions, Comm. Math. Phys., 163 (1994), pp. 245-255.

[To4] A. TovBIS, Breaking homoclinic connections for a singularly perturbed differential equation and the Stokes phenomenon, Stud. Appl. Math., 104 (2000), pp. 353-386.

[To5] A. TovBIS, On approximation of stable and unstable manifolds and the Stokes phenomenon, in Nonlinear PDE's, Dynamics and Continuum Physics, Contemp. Math. 255, AMS, Providence, RI, 2000, pp. 199-228.

[TP] A. Tovbis And D. Pelinovsky, Exact conditions for existence of homoclinic orbits in the fifth-order KdV model, Nonlinearity, 19 (2006), pp. 2277-2312.

[WW] E. T. Whittaker and G. N. Watson, A Course of Modern Analysis, Cambridge University Press, Cambridge, UK, 1927.

[Wa] W. WASOW, Asymptotic Expansions for Ordinary Differential Equations, Robert E. Krieger Publishing, Huntington, NY, 1976.

Copyright (c) by SIAM. Unauthorized reproduction of this article is prohibited. 